\title{
Carbon Nanotube Films: Preparation and Application in Flexible Electronics
}

\author{
WANG Haomin ${ }^{1,2}$, HE Maoshuai ${ }^{1,3,{ }^{*}}$, ZHANG Yingying ${ }^{2, *}$ \\ ${ }^{1}$ School of Materials Science and Engineering, Shandong University of Science and Technology, Qingdao 266590, \\ Shandong Province, P. R. China. \\ ${ }^{2}$ Department of Chemistry, Tsinghua University, Beijing 100084, P. R. China. \\ ${ }^{3}$ College of Chemistry and Molecular Engineering, Qingdao University of Science and Technology, Qingdao 266042, \\ Shandong Province, P. R. China.
}

\begin{abstract}
Flexible electronic devices have attracted immense attention in recent years. Conventional electronics that are predominantly fabricated with rigid metallic materials demonstrate poor flexibility. Compared to traditional electronic devices, flexible electronic devices with better flexibility can adapt to different working environments. Consequently, they fit perfectly with different systems with minimal rejections. However, such flexible electronic devices need to achieve good extensibility and flexibility without compromising on their electronic properties. Therefore, new challenges and requirements arise while

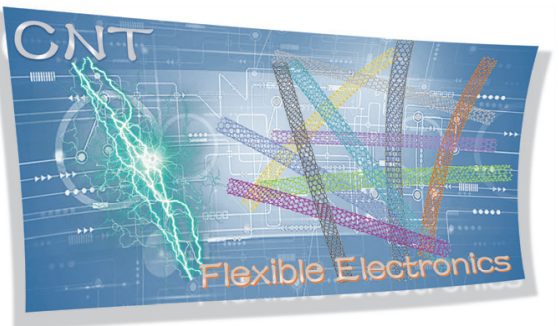
fabricating conductive materials. Manufacturing of flexible metal electrodes for flexible electronic devices include strategies such as reducing the thickness of the electrodes and designing electrodes with unique structures. However, these technologies are complex and expensive. Carbon nanotube (CNT) films exhibit good flexibility, excellent conductivity, good chemical and thermal stability, as well as good optical transparency, making them ideal candidates for flexible electronics. Therefore, the preparation and application of CNT films for the development of next generation flexible electronics have been extensively studied. In this review, we summarize the recent advances in the preparation of CNT films and their application in flexible electronic devices. Initially, the two main kinds of preparation methods for CNT films-dry and wet methods-are introduced. The dry methods for CNT film preparation include the membrane extraction method based on a vertical array of CNTs and the floating catalytic chemical vapor deposition method. Moreover, the wet methods predominantly discussed include vacuum filtration method, impregnation method, electrodeposition method, self-assembly method, and spraying method. Subsequently, the latest research advancements in assembly techniques, their performance and applications in various flexible electronics are discussed. This review primarily introduces the application of CNT films in the fields of flexible sensors, flexible energy devices, flexible transistors, and flexible display screens. The fundamentals of typical flexible sensors, such as strain sensors, pressure sensors, gas sensors, temperature sensors, and humidity sensors are presented. Besides, flexible lithium-ion batteries, flexible nanogenerators, and flexible thermoelectric devices based on CNT films are also investigated. Moreover, other flexible electronic devices, such as flexible transparent conductive film, flexible transistor, and flexible photodetector, based on CNT films are briefly described. Finally, advanced flexible electronics based on CNT films are summarized. The challenges and future prospects of these films are also discussed.
\end{abstract}

Key Words: Carbon nanotube film; Dry method; Wet method; Conductive film; Flexible electronics

\footnotetext{
Received: November 8, 2018; Revised: November 30, 2018; Accepted: December 3, 2018; Published online: December 7, 2018.

${ }^{*}$ Corresponding authors. Emails: hemaoshuai@qust.edu.cn (H.M.); yingyingzhang@tsinghua.edu.cn (Z.Y.). Tel.: +86-13864807842 (H.M.);

$+86-10-62798503$ (Z.Y.).

The project was supported by the National Natural Science Foundation of China (51672153) and the National Key Basic Research Program of China (973)

(2016YFA0200103).

国家自然科学基金(51672153)和国家重点基础研究发展规划项目(973) (2016YFA0200103)资助

(C) Editorial office of Acta Physico-Chimica Sinica
} 


\title{
碳纳米管薄膜的制备及其在柔性电子器件中的应用
}

\author{
王影珉 1,2 ，何茂帅 $1,3,{ }^{*}$ ，张芗芗 ${ }^{2}{ }^{*}$ \\ 1 山东科技大学材料科学与工程学院, 山东青岛 266590 \\ 2 清华大学化学系, 北京 100084 \\ 3 青岛科技大学化学与分子工程学院, 山东青岛 266042
}

摘要: 近年来, 柔性电子器件的发展日新月异。以碳纳米管为代表的碳纳米材料, 尤其是其组装成的宏观结构碳纳米管薄膜 具有良好的柔性和优异的导电性, 且具有化学稳定、热稳定、光学透明性等优点, 在柔性电子领域展现了极大的应用潜力。 本文简要综述了近年来碳纳米管薄膜在柔性电子器件领域的研究进展。首先详细介绍了碳纳米管薄膜的两类主要制备方法, 分别为干法制备和湿法制备; 继而介绍了碳纳米管薄膜在多种柔性电子器件的组装、性能与应用方面的最新研究进展; 最后 总结了碳纳米管薄膜基柔性电子领域的发展现状，并讨论了该领域所面临的挑战及其未来前景。

关键词：碳纳米管薄膜；干法制备；湿法制备；导电薄膜；柔性电子 中图分类号：0649

\section{1 引言}

近年来, 柔性电子器件因其在可穿戴表皮电 子与可植入生物医疗器件中的巨大潜在应用, 使 得该领域的发展日新月异 ${ }^{1-6}$ 。相对于传统电子器 件, 柔性电子器件具有更大的灵活性, 能够在一定 程度上适应不同的工作环境，与生物体完美贴合， 从而可以最大限度地减少生物体排斥, 但是相应 的技术要求同样制约了柔性电子器件的发展。首 先, 柔性电子器件需要在不损坏本身电子性能的 基础上获得伸展性和弯曲性, 对导电材料提出了 新的挑战和要求; 其次, 柔性电子的制备条件以及 组成电路的各种电子器件的高性能和稳定性相对 于传统的电子器件来说仍然不足, 也是其发展的 一大难题。在传统电子器件中的电极材料以金属 为主, 由于金属不具有柔性, 在拉伸或弯曲过程中 易于发生断裂而失效, 无法满足柔性器件的发展 需求。所以, 具有柔性的且导电性能可与传统金属 相媲美的材料的发展变得至关重要。目前, 被广泛 研究的柔性材料有: 水凝胶 7,8 、液态金属 9 、高分子 聚合物 ${ }^{10}$ 以及导电纳米材料 11,12 。水凝胶以高分子 网络为骨架, 网络中充满水分, 生物相容性强, 容 易掺杂具有导电性, 并且可以像人体皮肤一样自
愈合 ${ }^{13}$; 液态金属是常温或者接近室温下呈现液态 相的金属, 具有很强的导电性; 高分子聚合物过掺 杂可以成为导体来用作导线，或者成为半导体用 来做有机场效应晶体管 ${ }^{10}$; 纳米材料(如金属纳米 材料, 碳纳米材料 $14-19$ 等)由于其尺寸小, 本身或者 与其他弹性体复合, 可具有一定的柔性以及可拉 伸性。在这里特别指出的是, 碳纳米材料中的碳纳 米管薄膜材料 $20-23$ 由于具有优异的导电性 $24-26$ 和导 热性 ${ }^{27,28}$, 出色的机械与化学稳定性 ${ }^{29-33}$, 良好的 柔性以及具有结构与性能易调控且易大面积制备 等特点, 近年来在柔性电子领域薮露头角, 得到了 广泛且飞速的发展, 可能给人们生活带来革命性 的变革。本文将着重阐述碳纳米管薄膜制备方法 方面的重要研究进展, 讨论其在柔性电子器件应 用领域的关键科学和技术问题, 总结并展望了碳 纳米管薄膜在柔性电子器件领域的应用。

\section{2 碳纳米管薄膜的制备}

1991年，日本NEC公司的饭岛澄男(Iijima) ${ }^{34}$ 用高分辨透射电镜观察到了碳纳米管并在Nature 杂志上进行了报道。碳纳米管(Carbon nanotube, CNT), 是由石墨片卷曲而形成的无缝中空管体。碳

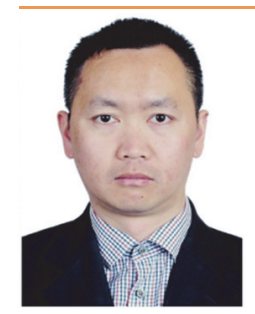

何茂帅，1980年生。青岛科技大学 化学学院教授, 博士生导师。2006 年于北京大学获得博士学位。2016 年加入山东科技大学。2018年起任 职于青岛科技大学。主要研究方向 为单壁碳纳米管的可控制备及其 生长机制探索。

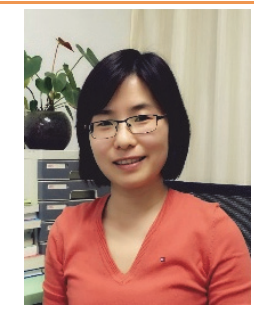

张荣荣, 1979年生。清华大学化学 系副教授, 博士生导师。2007年于 北京大学获得博士学位, 2008年 至2011年在美国洛斯阿拉莫斯国 家实验室从事博士后研究。2011 年起任职于清华大学。主要研究 方向为纳米碳材料与丝蛋白材料的制备、性能及 其在柔性可穿戴器件中的应用。 
纳米管中的碳原子以 $s p^{2}$ 方式进行杂化成键, 以六 元环为基本结构单元, 这使得碳纳米管具有很高 的杨氏模量, 是具有高断裂强度的材料, 在弯曲情 况下不容易损坏 ${ }^{35-38}$ 。碳纳米管还具有高的电导率 和高的热导率, 此外其可以通过制备工艺调控, 而 呈现半导体性或金属性。碳纳米管具有极大的长径 比、优异的机械强度以及良好的导电导热能力, 是 一种理想的柔性导电材料。碳纳米管的制备方法 主要有三种, 分别是电弧放电法(arc-discharge) ${ }^{39-42}$ 、 激光烧蚀法(laser ablation) ${ }^{43-46}$ 和化学气相沉积法 (chemical vapor deposition, CVD) ${ }^{47,48}$ 。而化学气 相沉积法由于其可控性强, 成本较低等优点, 已经 成为了制备碳纳米管的主流方法。在实际应用中, 碳纳米管又有多种形貌, 包括碳纳米管粉体 ${ }^{40}$ 、碳 纳米管纤维 ${ }^{49}$ 、碳纳米管薄膜 ${ }^{19}$ 、碳纳米管海绵、碳 纳米管垂直阵列 (VACNT) ${ }^{50,51}$ (又称为碳纳米管森 林)、碳纳米管水平阵列(HACNT) ${ }^{52,53}$ 等, 它们均 可用于柔性电子器件。本节将主要综述碳纳米管 薄膜的两大类主要制备方法, 包括干法制备和湿 法制备方法。

\section{1 碳纳米管薄膜的干法制备}

碳纳米管薄膜的干法制备是指薄膜制备的整 体过程都不接触液体, 主要有以下两种方法, 一种 是从碳纳米管的垂直阵列中直接抽出碳纳米管薄 膜, 另外一种是通过 $\mathrm{CVD}$ 的方法直接生长出碳纳 米管的薄膜。

\section{1 .1 抽膜法}

碳纳米管薄膜可以从碳纳米管垂直阵列中直 接抽取而获得。2002年, 清华大学Jiang等 ${ }^{49}$ 首次成 功从碳纳米管阵列中抽丝得到连续碳纳米管长 线。碳纳米管之间由于范德华力的作用从而可以 首尾相连得以连续地被抽出来, 他们从硅基底上 的超顺排碳纳米管阵列直接抽出长达 $30 \mathrm{~cm}$ 的碳 纳米管线。2005年, Baughman研究组 ${ }^{54}$ 在Science杂 志上报道了从碳纳米管垂直阵列中连续地抽出碳 纳米管透明薄膜, 薄膜长度可达米级。为了能够实 现这类碳纳米管薄膜的大规模生产和应用, 需要 制备出大尺寸的超顺排的碳纳米管垂直阵列。随 后, 清华大学范守善研究组 ${ }^{55}$ 在直径为 $20.32 \mathrm{~cm}$ 硅 基底上成功制备得到了碳纳米管垂直阵列, 实现 了该类碳纳米管垂直阵列以流水线的方式大量制 备, 并结合传统的干法纺丝技术, 得到了高度取向 的连续碳纳米管薄膜, 该薄膜长达 $300 \mathrm{~m}$ (图1a, b)。该直接抽出的碳纳米管透明薄膜有着优异的 导电性和良好的柔性, 而且能够自由控制形成交 叉堆叠结构 ${ }^{56}$ (图 $1 \mathrm{c}, \mathrm{d}$ ), 从而有利于广泛地应用 在柔性电子领域。

\section{1 .2 浮动催化化学气相沉积法}

碳纳米管薄膜还可以通过浮动催化化学气相 沉积法(FCCVD)的方法得到。2007年, 中国科学院 物理研究所解思深教授研究组 ${ }^{57}$ 通过浮动催化化 学气相沉积技术在 $5 \mathrm{~cm} \times 10 \mathrm{~cm}$ 的基底上生长出均 匀的碳纳米管薄膜 (图 $2 a-c)$, 该薄膜具有高电导 率、高透明度。制备过程中, 通过载气在 $65-85^{\circ} \mathrm{C}$
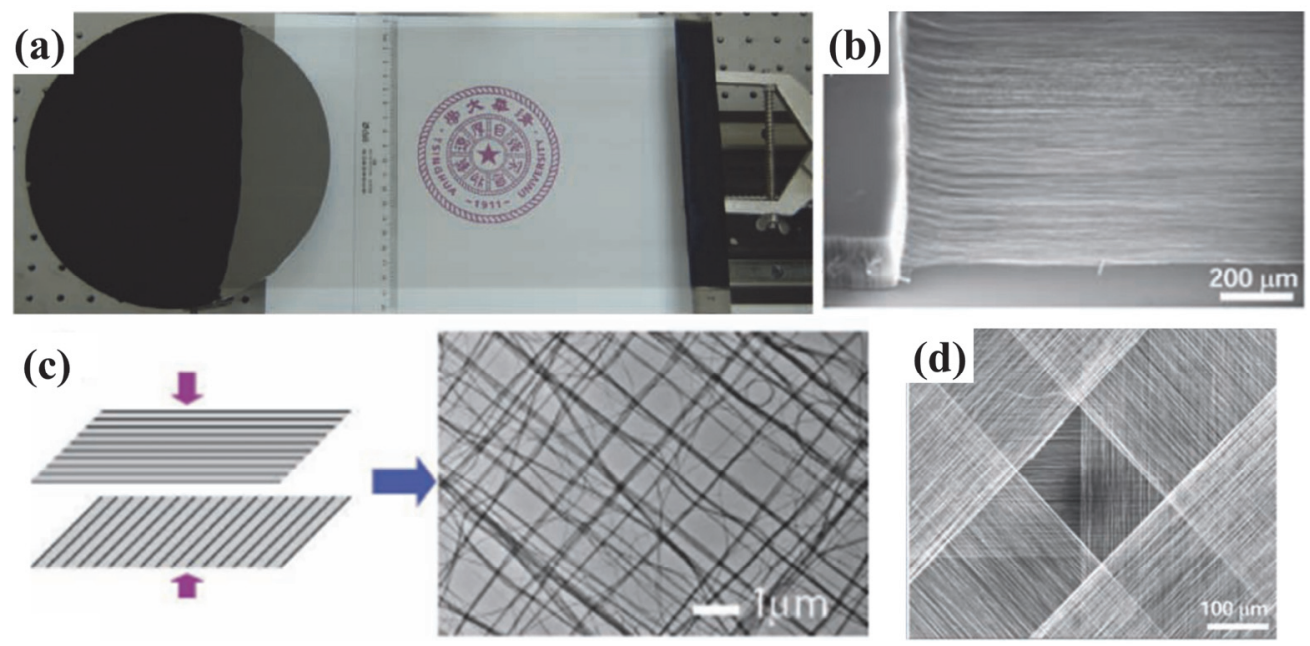

图 1 从碳纳米管垂直阵列(VACNT)直接抽丝成碳纳米管薄膜

Fig. 1 Directly spun carbon nanotube film from vertical array of carbon nanotubes (VACNT).

(a) optical image of carbon nanotube film from the VACNT ${ }^{56}$; (b) Scanning electron microscopy (SEM) image of carbon nanotube film from the VACNT ${ }^{54}$;

(c) Schematic illustration of making superaligned cross-stack films and a transmission electron microscopy (TEM) image ${ }^{56}$;

(d) SEM micrograph of a two-dimensionally reinforced structure fabricated by overlaying four carbon nanotube sheets ${ }^{54}$ 

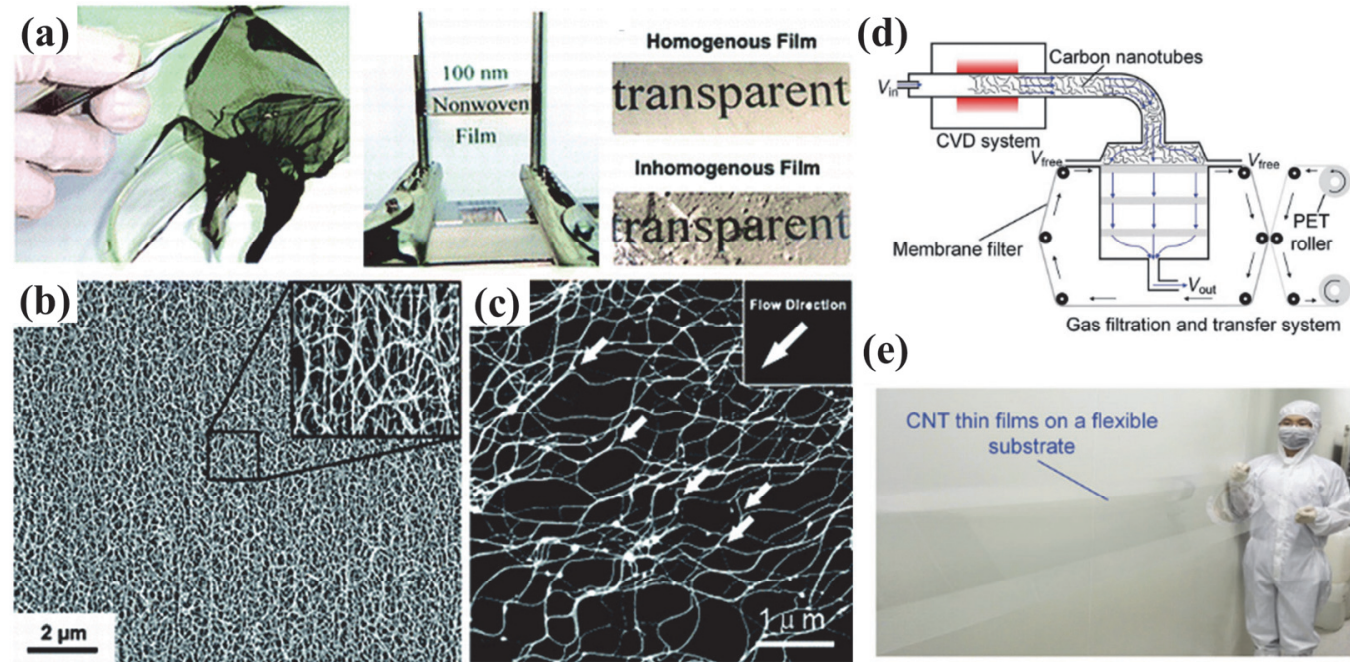

(e)

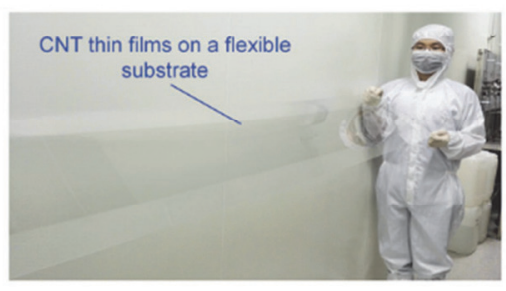

图 2 浮动催化化学气相沉积法(FCCVD)直接制备碳纳米管薄膜

Fig. 2 CNT film directly prepared through FCCVD (floating catalytic chemical vapor deposition method).

(a) A free-standing CNT film directly prepared through FCCVD and (b, c) its SEM image showing the alignment of CNT bundles ${ }^{57}$;

(d) Schematic showing the apparatus designed for the synthesis, deposition, and transfer of CNT films ${ }^{58}$;

(e) A CNT thin film transferred on a flexible PET (polyethylene terephthalate) substrate with a length of more than $2 \mathrm{~m}^{58}$.

的条件下将二茂铁/硫粉的混合物送入反应区域, 在 $600{ }^{\circ} \mathrm{C}$ 的条件下以甲烷为碳源制备碳纳米管薄膜, 生长 $30 \mathrm{~min}$ 薄膜的厚度可达 $100 \mathrm{~nm}$ 。制备得到的碳 纳米管薄膜可以轻易揭下, 转移至其他基底。2018 年, 中国科学院金属研究所成会明研究团队 ${ }^{58}$ 采用 浮动催化剂化学气相沉积方法在反应炉的高温 $\left(1100^{\circ} \mathrm{C}\right)$ 区域连续生长单壁碳纳米管 $(\mathrm{SWNT})$, 然 后通过气体过滤和转移系统在室温下收集所制备 的碳纳米管。当微孔滤膜沿着过滤腔室的四周移 动时, 可以在其上连续沉积大面积碳纳米管薄膜, 并且制备的碳纳米管薄膜可通过卷到卷滚压转移 方式转移至柔性聚对苯二甲酸乙二酯(PET)基底 上, 获得了长度超过 $2 \mathrm{~m}$ 的成卷碳纳米管薄膜(图 $2 \mathrm{~d}, \mathrm{e})$ 。薄膜具有优异的光电性能, 在 $550 \mathrm{~nm}$ 波长 下其透光率为 $90 \%$, 方块电阻 $65 \Omega \cdot \mathrm{sq}^{-1}$, 这为碳纳 米管薄膜的实际应用奠定了基础, 率先实现了大 面积、高均匀性SWNT薄膜的连续制备与转移。

\section{2 碳纳米管薄膜的湿法制备}

碳纳米管薄膜的湿法制备主要指对制备得到 的碳纳米管样品 (一般为粉体样品)在溶剂中进行 纯化、分散、分离, 然后通过一定的方法将其制备 成碳纳米管薄膜。该方法的优势在于碳纳米管在 溶剂中处理的过程中能够最大限度地去除无用的 杂质, 这更利于得到有着更高导电性和更高纯度 碳纳米管薄膜, 甚至可以在溶剂中对其进行手性 的选择, 从而制备不同性质(金属性/半导体性)的 碳纳米管薄膜, 对其应用有着很重要的意义。具体
而言, 通过湿法制备碳纳米管薄膜的方法又可分 为抽滤法、浸渍法、电沉积法、自组装法和喷涂法 等。

\subsection{1 抽滤法}

抽滤法是制备各种薄膜材料最常用的方法之 一。起初, 研究者们主要借鉴以前抽滤纳米纤维膜 的方法, 简单的来抽滤碳纳米管溶液制作碳纳米 管薄膜 ${ }^{59}$ 。在 2004 年, Rinzler等 ${ }^{60}$ 首次报道了用真 空抽滤法制备碳纳米管薄膜。研究者首先用十二 烷基硫酸钠(SDS)水溶液分散碳纳米管, 通过超声 获得在水中均匀分散的碳纳米管溶液, 再通过真 空抽滤的方法使碳纳米管沉积在滤膜上形成薄 膜, 在用去离子水冲洗后, 得到干净的碳纳米管薄 膜。这种薄膜展现出优异的导电性和透光性(图 3a)。然而, 上述这种方法制备得到的碳纳米管薄 膜就像抽滤得到的纳米纤维膜一样, 其中的碳纳 米管是随机分散搭接的, 不具备定向性。2016年, 莱斯大学Kono研究组 ${ }^{61}$ 改进了真空抽滤的方法, 通过表面带负电荷的表面活性剂增强碳纳米管间 的排斥力, 使碳纳米管在溶液中得以更均匀的分 散, 同时降低碳纳米管悬浊液浓度和抽滤速度得 到了高度定向的碳纳米管薄膜。通过调控实验参 数, 这种慢真空抽滤的方法可以控制薄膜厚度从 几纳米到几百纳米, 且碳纳米管的密度为 $10^{6}$ lines $\mu \mathrm{m}^{-1}$ 。

\subsection{2 浸涂法}

浸涂法是将基底浸入碳纳米管分散液中使碳 
(a)

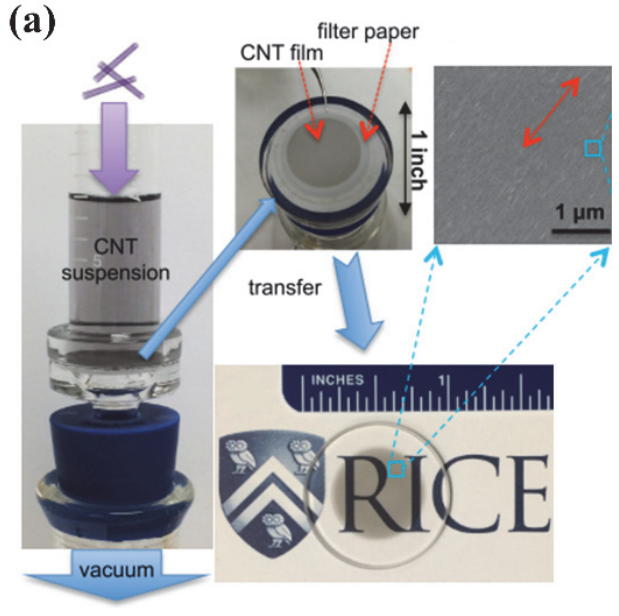

(b)

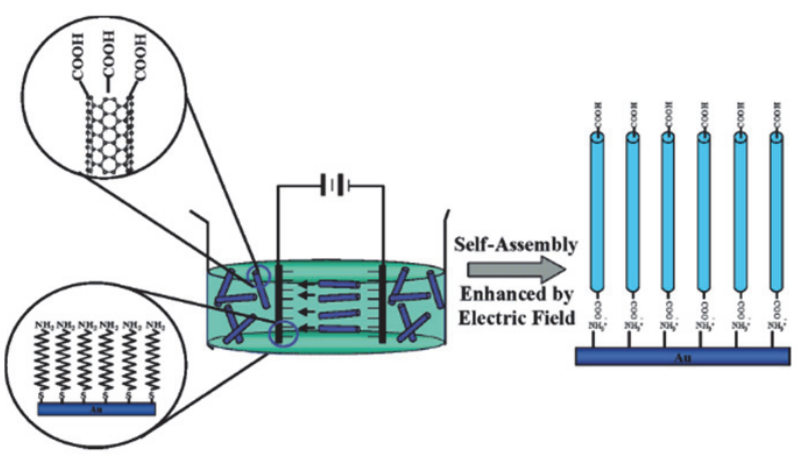

图 3 抽滤法和电沉积法制备碳纳米管薄膜

Fig. 3 Vacuum filtration and electrodeposition method for preparing CNT film.

(a) Preparation and Characterization of CNT film by vacuum filtration ${ }^{61}$; (b) Schematic illustration of the electric-field-enhanced chemical assembly of single-walled carbon nanotubes on a gold surface ${ }^{64}$.

纳米管吸附在基底上，一段时间后将其从溶液中 拿出, 待干燥后自然成膜从而得到连续的碳纳米 管薄膜的方法。Lima等 ${ }^{2}$ 最初以单壁、双壁或多壁 碳纳米管为原料添加表面活性剂制备分散均匀的 碳纳米管水溶液, 然后采用浸涂法用基底浸入该 溶液中制备碳纳米管薄膜, 且发现得到的薄膜表 面相对较为光滑, 且该碳纳米管薄膜可从原始基 底上转移到其他基底, 该碳纳米管薄膜在 $550 \mathrm{~nm}$ 波长下透光率为 $94 \%$, 方块电阻为 $3.6 \mathrm{k} \Omega \cdot \mathrm{sq}^{-1}$ 。Poa 等 ${ }^{63}$ 将基底浸入氨丙基三乙基氧硅烷(APTS)的水 溶液中, 使基底表面上涂覆一层APTS分子, 并在 氮气气氛下干燥, 然后浸入用Triton X-100分散的 SWNT水溶液中 $2 \mathrm{~min}$ 后并缓慢提出。通过这种方 法, 他们得到了室温下大面积涂覆于玻璃或塑料 基底上的碳纳米管薄膜。经过硝酸处理后, 得到的 碳纳米管薄膜方块电阻约为 $130 \Omega \cdot \mathrm{sq}^{-1}$, 透光率为 $69 \%$, 并且通过控制浸渍的次数, 可以很容易地调 整薄膜的透明度和薄膜电阻。

\subsection{3电化学沉积法}

电沉积法也是很有潜力的制备碳纳米管薄膜 的工艺之一, 该方法由 Guo等 ${ }^{64}$ 首先使用, 通过电 场实现了排列规则的碳纳米管薄膜的制备(图3b), 且电场越强, 成膜速度将会越快。随后在2009年, 成会明教授课题组 ${ }^{65}$ 采用先电位沉积, 随后对其进 行热压的工艺, 制备出方块电阻为 $220 \Omega \cdot \mathrm{sq}^{-1}$ 、透 光率为 $81 \%$ 的薄膜, 并且具有良好的柔性和稳定 性, 在弯折过程中其导电性不受影响。这类方法得 到的碳纳米管薄膜均匀、光洁, 成膜速度快且可反 复利用, 可得到多种形状的薄膜。

\section{2 .4 自组装法}

自组装(self-assembly) 是指基本结构单元(单 根碳纳米管)自发形成有序结构的一种技术, 该技 术基于整体的复杂的协同作用。在2008年, 美国 IBM研究中心Engel等 ${ }^{66}$ 利用黏滑(Slip-stick)机制 将硅片基底放入用SDS (质量分数 $1 \%$ )分散的碳纳 米管溶液中, 类似 “咖啡环” 的现象, 在干燥后自 组装了碳纳米管的超晶格顺排结构薄膜(图4a, b), 碳纳米管的密度达到 20 lines $\mu \mathrm{m}^{-1}$ 。同年, Xie等 ${ }^{67}$ 使用两亲分子对碳纳米管进行化学修饰, 并使用 LB (Langmuir-Blodgett)膜法将其加工成膜。他们 发现碳纳米管的长度为 $1-2 \mu \mathrm{m}$ 时可以得到定向性 良好的碳纳米管, 碳纳米管过长或太短都会影响 碳纳米管的定向排列。使用该方法得到大约为 18 层的薄膜, 其透光率可以达到 $93 \%$ 。2012年, 美国 IBM研究中心Park等 ${ }^{68}$ 采用一种离子交换表面化 学处理方法实现了高密度碳纳米管的定位(图4c), 碳纳米管被选择性地沉积在经过表面处理的 $\mathrm{HfO}_{2}$ 区域内。在对使用该方法制备的晶体管进行检测 后, 发现其中 $90 \%$ 的器件都表现出良好的电学接 触, 而且 $80 \%$ 的晶体管具有半导体性的开关特性。 在 2013 年, 该中心的 Cao 等 ${ }^{69}$ 报道了采用 LS (Langmuir-Schaefer)成膜技术将纯度为 $99 \%$ 的半导 体型碳纳米管排列成高密度顺排碳纳米管薄膜 (图4d), 该薄膜一般由两层碳纳米管构成, 碳纳米 管的密度达 500 lines $\mu \mathrm{m}^{-1}$, 考虑到碳纳米管直径的 因素, 该密度已经接近于理论极限, 进一步地, 使 用这种碳纳米管薄膜加工而成的晶体管, 其电导 率高于 $40 \mu \mathrm{S} \cdot \mu \mathrm{m}^{-1}$, 开关比可达 $10^{3}$ 量级。2014年, 

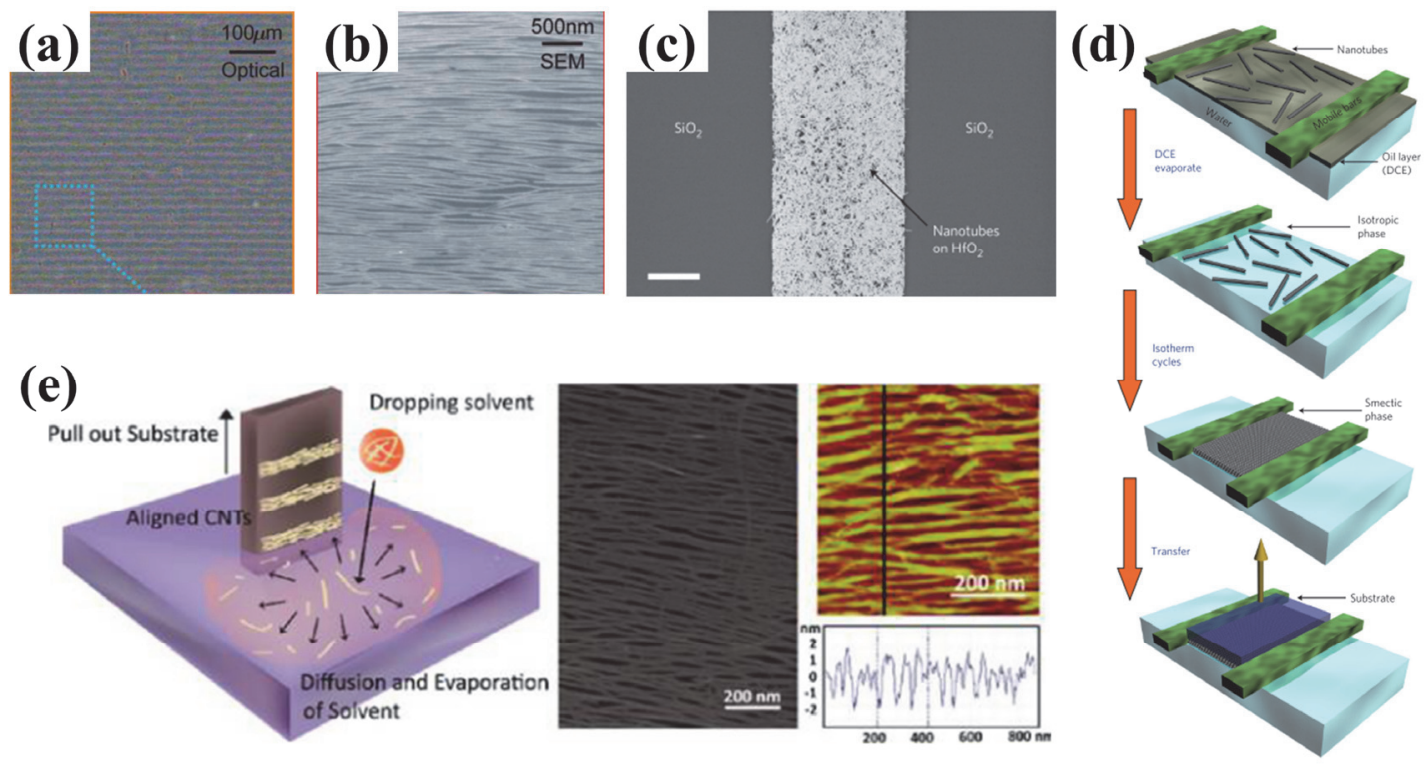

图 4 自组装法制备碳纳米管薄膜及其表征

Fig. 4 Preparation and Characterization of CNT film by self-assembly method.

(a) Optical micrograph and (b) SEM image showing the large scale alignment of the nanotube superlattice ${ }^{66}$; (c) SEM image of CNTs deposited on an open $\mathrm{HfO}_{2}$ area ${ }^{68}$; (d) Schematic illustration of the Langmuir-Schaefer assembly process ${ }^{69}$; (e) Preparation and Characterization of CNT film by a novel "dose-controlled, floating evaporative self-assembly" process ${ }^{70}$.

Joo 等 ${ }^{70}$ 开发了 “剂量控制, 浮动蒸发自组装” 的方 法(图4e)。首先将基底垂直浸入水中, 在靠近基底 的位置逐滴滴加溶解于有机溶剂中的碳纳米管溶 液, 同时逐渐向上提拉基底。由于一滴碳纳米管溶 液中包含的碳纳米管有限, 需要碳纳米管溶液的 不断滴入, 随后液面上会形成一条条顺排的碳纳 米管薄膜带。该工艺对沉积的半导体碳纳米管的 条纹位置和数量有很好的控制, 这种方法得到的 碳纳米管密度大约为 50 lines $\mu \mathrm{m}^{-1}$ 。

\section{2 .5 喷涂法}

喷涂法是指将碳纳米管分散液直接喷于基底 上的工艺, 其优点在于成膜效率高, 适合制备大面 积薄膜, 而且通过控制溶液的浓度、喷涂流量及喷 涂时间能够对薄膜厚度实现良好的调控 ${ }^{71-77} 。 2007$ 年, Lee课题组 ${ }^{73}$ 使用喷涂法在平整基底上制备了 碳纳米管薄膜, 并且在此基础上发现, 酸处理可以 显著改善薄膜的导电性能, 并成功制得了透光率 为 $80 \%$ 、方块电阻为 $70 \Omega \cdot \mathrm{sq}^{-1}$ 的碳纳米管薄膜。随 后, 该小组将正丙醇水溶液和全氟磺酸化树脂的 混合溶液用来分散SWNT, 将分散液喷涂在聚对苯 二甲酸乙二醇酯(PET)基底上, 通过 $p$ 型掺杂使得 碳纳米管薄膜的导电性得到提高, 最终得到了方 块电阻 $100 \Omega \cdot \mathrm{sq}^{-1}$ 、透光率为 $80 \%$ 的薄膜。随后, 喷 墨打印技术由于不需经过光刻就能制备出各种复 杂图案, 并且具有对材料的利用率高等优点, 近几
年来得到了飞速的发展 ${ }^{17,78}$ 。所以, 研究者将碳纳 米管加工成 “墨水” ，通过打印的方法可以在纸 上得到更为复杂的形状。清华大学魏飞教授课题 组 ${ }^{79}$ 用喷墨打印机将多壁碳纳米管分散液打印在 纸上, 得到了导电性能良好且与纸结合力牢固的碳 纳米管薄膜。 $\mathrm{Li}^{\text {等 }}{ }^{74}$ 通过喷墨打印技术将聚乙烯醇 功能化的 SWNT制成了方块电阻仅为 $225 \Omega \cdot \mathrm{sq}^{-1}$ 的 薄膜, 为实现低成本的电子产品应用提供了机会。 然而上述的碳纳米管喷涂油墨都需要表面活性剂 来使得碳纳米管稳定分散, 从而牺牲了制得的薄膜 的电学性能。在 2015 年, 胡良兵课题组 ${ }^{80}$ 发明了一 种无表面活性剂碳纳米管油墨, 适用于导电率达 到 $2100 \mathrm{~S} \cdot \mathrm{cm}^{-1}$ 的喷涂薄膜(图 $5 \mathrm{a}, \mathrm{b}$ )。对碳纳米管 油墨Zeta电位的测量直接证明了与喷涂膜导电性 呈正相关, 这是高性能印刷电子产品的关键指标。

湿法制备技术的关键是碳纳米管的分散问 题, 它决定碳纳米管薄膜的成膜质量及最终薄膜 的透明性及导电性。因此, 分散剂的选择对制备高 稳定性的分散液至关重要。虽然高极性的有机溶 剂如 $N, N$-二甲基甲酰胺(DMF)或 $N$-二甲基吡咯烷 酮(NMP)等对碳纳米管有良好的溶解性, 但出于实 际生产应用考虑, 更多的时候还是希望使用更加 安全的水溶液为溶剂。在水溶液体系中, 常使用表 面活性剂来对碳纳米管来进行有效的分散, 但是 使用这种碳纳米管分散液制备的碳纳米管薄膜由 


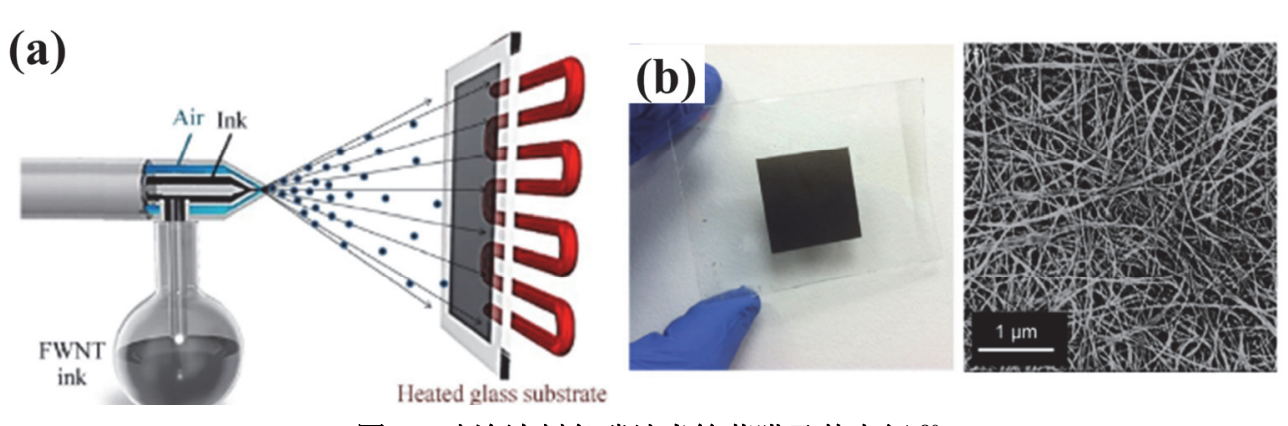

图 5 喷涂法制备碳纳米管薄膜及其表征 ${ }^{80}$

Fig. 5 Preparation and Characterization of CNT film by spray-coating ${ }^{80}$.

(a) A schematic depicting the fabrication process for the spray-coated CNT films; (b) Optical image and SEM image of spray-coated thin CNT films from a surfactant-free carbon nanotube ink.

于表面活性剂的影响, 常常使得薄膜的导电性变 差。而且在水溶液中使用表面活性剂分散时, 都离 不开超声分散, 而碳纳米管在强超声破碎的作用 力下, 常常会因此产生大量缺陷甚至发生断裂, 这 也严重影响了后续的应用。因此, 如何制备有着稳 定分散且碳纳米管保持完好的安全的分散液也是 目前该领域面临的问题。

\section{3 碳纳米管薄膜在柔性电子器件中的应用}

碳纳米管薄膜由于其本身具有出色的导电 性、导热性、机械稳定性和良好的柔性, 使其能够 柔性电子器件领域都有着广泛的应用, 比如: 柔性 传感器、柔性能源器件, 柔性导电薄膜材料等。下 面将分别概述其在柔性电子器件中的应用及发 展。

\section{1 碳纳米管薄膜基柔性传感器}

柔性传感器是指采用柔性材料制成的传感 器, 具有良好的柔韧性、延展性、甚至可自由弯曲 甚至折叠, 而且结构形式灵活多样, 可根据测量条 件的要求任意布置, 能够非常方便地进行检测, 它 是监测人体生物医学信号、运动和环境的理想传 感器 ${ }^{81-83}$ 。柔性传感器通常由电极、敏感材料和和 柔性基底组成, 特别是合适的敏感材料的选择对 传感器的性能起着举足轻重的作用。碳纳米管薄 膜材料由于其卓越的机械性能、导电性和导热性, 以及制备方法多样, 结构可控等特点, 被广泛应用 于柔性传感领域。

\section{1 .1 应变传感器}

柔性应变传感器可以将感受到的应变信号转 化为电学等信号, 从而能够对应变产生响应。它可 以实时监测人体各处的大大小小的形变如脉搏振 动, 声带振动, 四肢运动等 $81,82,84$ 。一种制备柔性 应变传感器优异的材料一碳纳米管薄膜, 它的电 学性质在发生应变时会发生改变 ${ }^{85-89}$ 。早在2004
年, Dharap等 ${ }^{90}$ 就利用这种碳纳米管的应变传感特 性, 研制了一种可用于宏观应变传感的碳纳米管 薄膜传感器。由于SWNT是随机分散在基底上的, 所以该碳纳米管薄膜是各向同性的。实验结果表 明, 在拉伸和压缩应力作用下, 薄膜的电压都是几 乎呈线性变化的。表明了这种薄膜在宏观尺度上对 多方向和多位置应变传感器的潜力。2011年，Hata 研究组 ${ }^{91}$ 报道了一种由顺排的单壁碳纳米管薄膜 制成的可穿戴和可拉伸传感器(图6a)。将单壁碳纳 米管垂直阵列切分成 $1 \mathrm{~mm}$ 的薄膜分别取出, 并排 排列垂直于应变轴放置于狗骨头形状的弹性体薄 膜上。当拉伸时, 碳纳米管薄膜断裂成缺口和岛 状，并形成束状的缺口。由于此机制的存在，这种 薄膜可以充当应变传感器，并能够测量高达 $280 \%$ 的应变(比传统的金属应变计高出50倍), 具有高耐 久性、响应迅速和低蠕变的特点。研究者在长袜 (图6b)、朋带和手套上装配了碳纳米管传感器, 以 制造出能够检测不同类型的人类运动的设备, 包 括运动、打字、呼吸和说话等。2015年, Lee研究 组 ${ }^{92}$ 制备了一种可拉伸的、透明的、超灵敏的应变 传感器, 它是由一种新型的灵敏纳米复合薄膜 (SWNT薄膜)和一种由聚氨酯(PU)-聚( 3,4 -乙二氧 基噻吩)聚苯乙烯磺酸酯(PEDOT:PSS)构成的导电 弹性体复合材料。当传感器附着在脸上的皮肤时, 有着高达 $100 \%$ 的拉伸性, 光学透明度为 $62 \%$, 且 应变系数达到了 62 。通过实验证实, 这种传感器可 以检测到人体皮肤上的小应力引起的情感表达，如 笑和哭, 以及眼球运动等。

\section{1 .2 压力传感器}

人体皮肤是最复杂的器官之一。它拥有一个 高度敏感的触觉传感器网络, 能够将机械刺激转 化为生理信号, 然后由大脑进行解析。所以为了模 拟人类的皮肤，电子皮肤(e-skin)由于其具有的广 泛的潜在应用, 在科学界引起了人们极大的兴趣 ${ }^{93}$ 。 
(a)

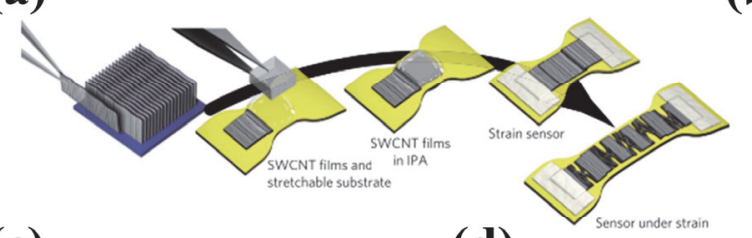

(c)

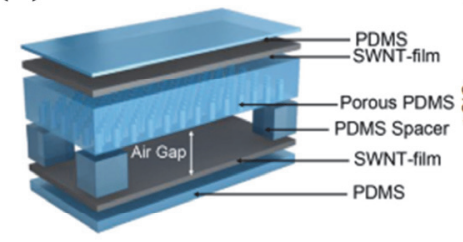

(f)

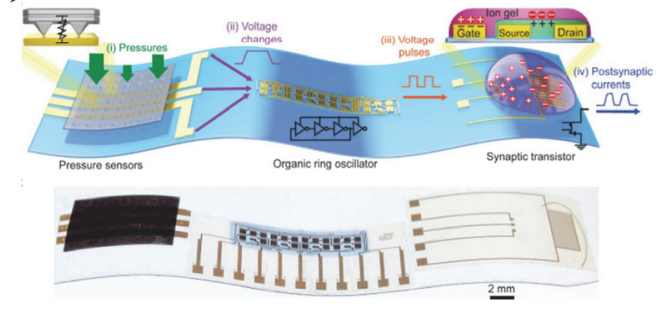

(g) (b)

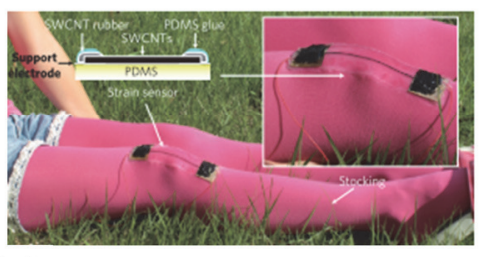

(e)
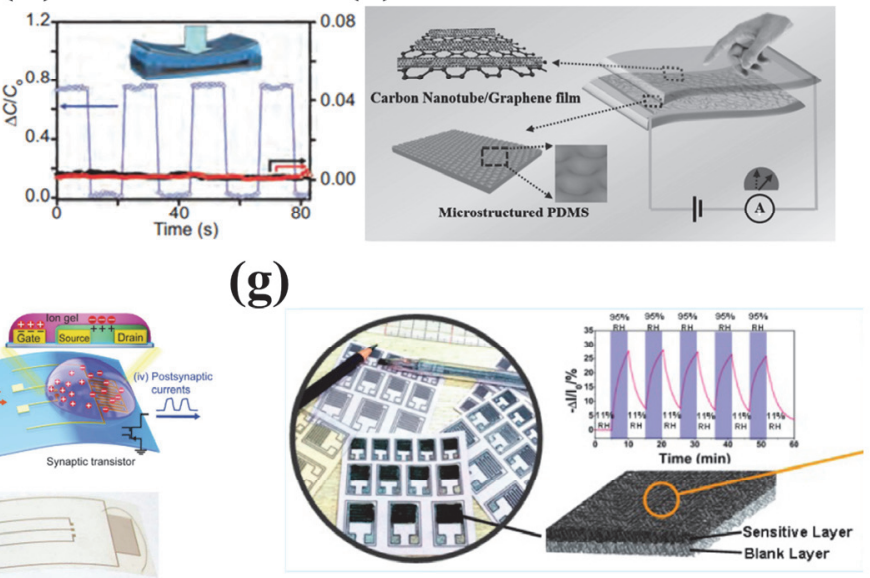

图 6 碳纳米管薄膜在多种柔性传感器中的应用

Fig. 6 Application of CNT films in various flexible sensors.

(a) Fabrication process of CNT film-based strain sensors and (b) flexible strain sensor attached to human knee joint for detecting the motions ${ }^{91}$;

(c) Schematic diagram of the pressure sensors based on porous PDMS (polydimethylsiloxane) and SWNT (single-walled carbon nanotube) films and

(d) the sensors for distinguishing pressure ${ }^{95}$; (e) Schematic illustration of the flexible pressure sensors made of CNT/graphene hybrid films and microstructured PDMS substrates ${ }^{97}$; (f) Schematic diagram and photograph of an artificial afferent nerve system ${ }^{96}$;

(g) Fabrication and performance of CNT-based humidity sensor ${ }^{105}$.

其中, 电子皮肤对压力的响应是极其重要的一部 分, 压力传感器可以将感受到的压力信号转变为 电学等信号94。2011年, Lipomi等88报道了透明的、 导电的单壁碳纳米管沉积的薄膜来用作压力传感 器。2014年, Bao等95 将碳纳米管分散在DMF溶液 中, 采用喷涂技术把碳纳米管混合物喷涂在预先 用等离子体处理过的聚二甲基硅氧烷(PDMS)基 底上。利用等离子体处理后的PDMS表面变成亲水 性更有利于碳纳米管的均匀分布。该复合物薄膜 受到外界机械力刺激时, 由于碳纳米管管间的接 触会发生变化, 从而伴随着电阻的变化, 由此得到 了电阻式压力传感器。这种传感器可以测量 $0.1 \mathrm{~Pa}$ 以上的机械刺激压力(图6c, d)。之后, 在2018年该 课题组 ${ }^{96}$ 又报道了一种基于柔性有机电子器件的 高灵敏度仿生触觉神经系统(图6f)。这种人造感知 神经由三个核心部件组成: 电阻式压力传感器、有 机环形振荡器、突触晶体管。其中碳纳米管薄膜充 当该压力传感器的一个电极。该电阻压力传感器 由导电金字塔结构的弹性体在碳纳米管薄膜电极 和金电极之间形成电阻通路, 压力的增加使得电
极间接触面积增加, 因此减少了碳纳米管薄膜电 极和金电极之间的电阻, 从而对压力产生响应, 其 灵敏度和工作范围与生物体相当。该系统首先利 用一系列感受器感知极为细微的压力, 并产生相 应的电压变化, 随后通过环形振荡器(人工神经纤 维)将电压变化转变为电脉冲信号。多个环形振荡 器得到的电信号被突触晶体管集成转变为突触电 流, 进而传递到下一级神经。这种人工神经触觉系 统具有良好的生物兼容性、柔性和高灵敏度, 在仿 生机器人、义肢感触等领域具有广阔的应用前景。 此外, 碳纳米管薄膜与其他薄膜材料 (如石墨烯) 复 合制作的压力传感器也被报道。Zhang课题组 ${ }^{77}$ 将 定向排列的碳纳米管带状薄膜与CVD生长的石墨 烯薄膜集成在有微结构的PDMS上(图6e), 然后将 两个这样的复合薄膜有面对面组装成压力传感 器。由于丰富的微观结构以及薄膜间的充分接触, 在 $0.3 \mathrm{kPa}$ 的压力下, 该压力传感器表现出高达 19.8 $\mathrm{kPa}^{-1}$ 的灵敏度。

\section{1 .3 气体传感器}

气体传感器是一种将某种气体体积分数转化 
成对应电信号的转换器。气体检测在空气质量监 测、矿井工程、健康医疗等领域具有重要影响, 一 直以来备受关注。碳纳米管作为中空管状一维纳 米材料, 具有丰富的孔隙结构、高表面吸附能力、 良好的导电性等性能, 能够检测氨气、二氧化氮、 二氧化碳、氢气等多种气体, 是制作气敏传感器敏 感膜的理想材料。Woo等 98 用电弧法制备出碳纳米 管并将其溶解于十二烷基硫酸钠溶液中, 再利用 多孔氧化铝(AAO)模板真空过滤该溶液, 形成碳纳 米管薄膜层, 然后将载有碳纳米管薄膜层的上述 模板置于PDMS透明基底上, 高温 $100^{\circ} \mathrm{C}$ 烘烤 $2 \mathrm{~h}$ 左 右, 取出 AAO模板得到柔性碳纳米管敏感层, 传 感器弯曲能力强 $\left(180^{\circ}\right.$ 弯曲后能完全恢复), 对氨气 有较好的响应。Lin等 99 采用可靠、可重复的转移过 程制备的高度均匀的碳纳米管薄膜来用作气体探 测的电阻网络。将此碳纳米管网络集成于聚酰亚 胺(PI)的柔性基底上在室温下, 当环境 $\mathrm{CO}_{2}$ 气体浓 度为 $800 \times 10^{-6}$ 时, 柔性气敏传感器的灵敏度为 $2.23 \%$ 。该传感器的响应速度和响应稳定性均较好。 3.1 .4 温度传感器

温度传感器是指能感受温度并转换成可用输 出信号的传感器。温度传感器作为电子皮肤(eskin)的重要传感元件之一, 研究者们也对使用碳 纳米管、石墨烯和导电聚合物等多种材料制备的 温度传感器进行了研究 ${ }^{100}$ 。尤其是碳纳米管薄膜 本身的电阻会随着温度的改变而改变, 且该薄膜 制备方法多样, 结构可控, 得到了广泛的研究。 Karimov等 101 制作了一种基于碳纳米管的柔性温 度传感器, 他们将碳纳米管沉积在一种具有粘性 的聚合物胶带上, 并将其封装形成管状。多壁碳纳 米管的直径在10-30 nm之间, 沉积的碳纳米管层 的厚度在300-430 $\mu \mathrm{m}$ 之间, 两个电极的间距和其 宽度分别在 4-6 mm 和3-4 mm之间。通过实验得出 这种传感器的电阻-温度关系: 当温度从 $20^{\circ} \mathrm{C}$ 升到 $70{ }^{\circ} \mathrm{C}$ 时, 传感器在 $20^{\circ} \mathrm{C}$ 时的直流电阻值为 $70^{\circ} \mathrm{C}$ 时的 1.4 倍。该研究团队还制作了基于碳纳米管薄 膜的温度传感器, 该传感器的电阻随温度的升高 而下降, 呈良好的线性变化。将这种温度传感器粘 于柔性基底上, 性能依旧非常稳定。

\section{1 .5 湿度传感器}

湿度传感器把空气中的湿度通过一定检测装 置, 测量到湿度后, 按一定的规律变换成电信号或 其他所需形式的信息输出, 用以满足用户需求。湿 度传感器已经在环境检测、仓库存储、工业生产、 气象检测等方面得到广泛应用, 成为当前最具实 用性的传感器之一 ${ }^{102}$ 。由于碳纳米管具有比表面
积大、吸附能力强、电学特性优异等特点, 而且其 良好的吸附能力使碳纳米管易于吸附水汽，可作 为湿敏材料, 所以以碳纳米管为敏感材料的湿度 传感器具有灵敏度高、响应速度快等优点, 是目前 湿度传感器研究的热点 ${ }^{103,104}$ 。Zhao 等 ${ }^{105}$ 报道了一 种在纸基上制备的全碳湿度传感器(图6g)。电极用 商用铅笔书写, 氧化多壁碳纳米管(O-MWCNTs) 油墨沉积成薄膜来当做敏感层。在动态测量中, 该 柔性湿度传感器具有良好的重复性和稳定性。这 种方法优化后的纸基传感器的响应比在传统陶瓷 基板上制作的传感器高5倍。在纸基的多孔表面分 散氧化多壁碳纳米管而形成的敏感层结构可以减 轻弯曲过程中的内应力。在极高的弯曲度下, 该柔 性纸基湿度传感器的响应仅有 $6.7 \%$ 的衰减。

\section{2 碳纳米管薄膜基柔性能源器件}

随着人们对生活质量的提高的需求, 柔性能 源器件在柔性和可穿戴电子器件等新兴领域已经 受到了广泛关注。新型的一维材料碳纳米管和二 维材料石墨烯具有非常优异的性能, 例如高比表 面积、稳定的热、化学性质以及高电导率等。其中, 碳纳米管薄膜的机械性能明显优于制备柔性能源 器件的其他材料, 它既可以直接作为柔性材料, 也 可以作为柔性材料的组分, 在此领域发挥着重要 作用。下面将分别介绍碳纳米管薄膜在柔性超级 电容器和柔性电池等储能领域以及在柔性热电器 件和柔性摩擦纳米发电机等发电领域的应用。

\section{2 .1 柔性超级电容器}

超级电容器是通过电极与电解质之间形成的 界面双层来存储能量的新型元器件。尽管储能器 件的应用非常广泛, 但是通常的制备方法不能由 脆性材料得到高柔性储能器件 ${ }^{106}$ 。碳纳米管的一 维结构具有极大的弯曲半径, 在极度弯曲条件下 仍然具有良好的电化学性能 107,108 。其次, 良好的 结合力使其经过多次弯曲之后还能紧密结合, 意 味着它们可以轻松形成独立的薄膜结构。因此, 碳 纳米管在柔性超级电容器领域发挥着重要作用。 Gruner等 109 发明了基于SWNT薄膜的全印刷高性 能超级电容器(图7a, b)。其中, 喷涂制备的单壁 碳纳米管薄膜作为活性材料用来同时当做电极和 电荷收集装置。这两个部分使用相同的材料来实 现, 消除了碳纳米管电荷收集器一金属电极间的 接触电阻, 从而实现了简化的、轻量级的体系结 构。单壁碳纳米管薄膜网络具有的高电流承载能 力和强大的机械强度使其可以满足可穿戴设备的 要求。由于单壁碳纳米管可以很容易的通过多种 方式分散在溶剂中制备成可打印的 “墨水”，因 

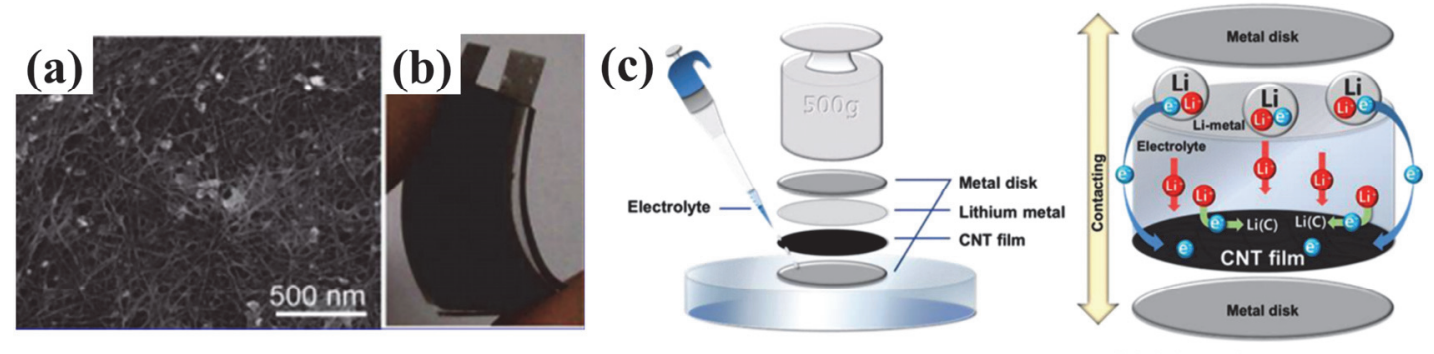

(d)
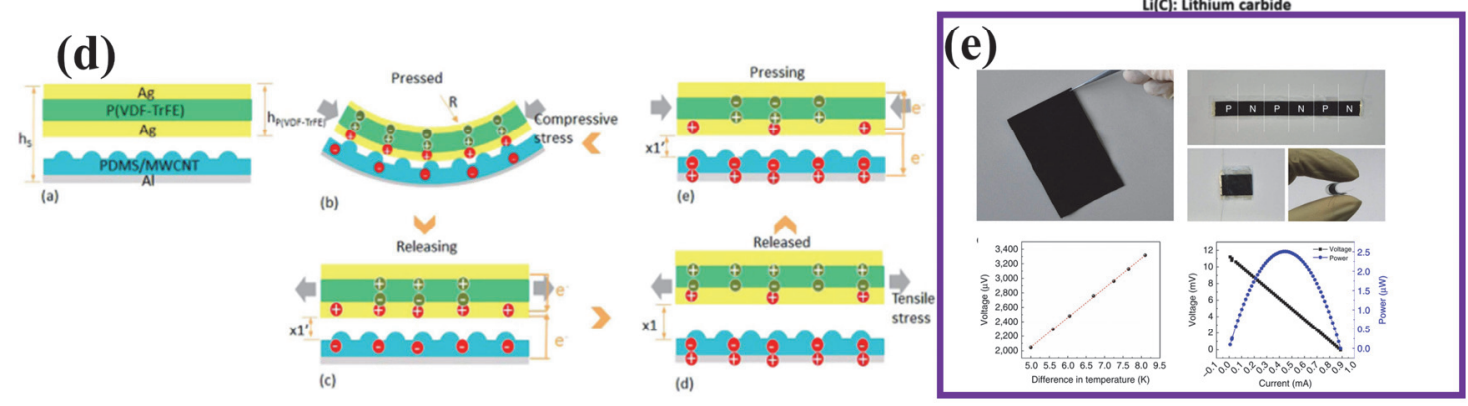

图 7 基于碳纳米管薄膜的各种柔性能源器件

Fig. 7 CNT films based various flexible energy device.

(a) SEM image of as-deposited SWCNT networks and (b) Thin film supercapacitor using sprayed SWNT films on PET as electrodes ${ }^{109}$; (c) Direct lithiation (DP) process of CNT film and schematic drawing of the principle of DP ${ }^{117}$; (d) Structure and working principle of the triboelectric and piezoelectric hybrid nanogenerator ${ }^{120}$. (e) Photographs and performance of compact-designed thermoelectric modules ${ }^{123}$.

此使用打印技术可以很容易地制备SWNT薄膜进 而制备出可印刷的柔性薄膜超级电容器。这种全 新的完全可打印的超级电容器在能量存储领域有 着极大的潜力 ${ }^{110,111}$ 。

\section{2 .2 柔性电池}

在柔性电池中, 碳纳米管主要用于可弯曲的 导电电极的制备 ${ }^{112,113}$ 。没有经过官能团化的碳纳 米管具有比官能团化的碳管更好的性能。碳纳米 管用于正负极材料的添加剂, 对降低电池的内阻 有极大的帮助。同时, 碳纳米管薄膜具有极大的比 表面积, 能够促进电解质的吸附性能进而促进离 子传输114,115。Chew等 ${ }^{116}$ 用抽滤的方法得到的多壁 碳纳米管薄膜来制备锂离子电池。实验证明多壁 碳纳米管薄膜电极具有显著的锂离子吸附和脱附 的潜力。Lee等 117 发现碳纳米管薄膜由于其固有的 性质, 导致其具有很大的初始不可逆电容, 从而会 导致锂离子电池的性能下降。所以, 他们将制备的 碳纳米管薄膜进行预锂化, 来降低初始不可逆电 容。将直接锂化(DP)法应用于CNT薄膜, 使其在电 池组装前与电解液中的锂金属发生反应(图7c)。将 预锂化的碳纳米管薄膜作为柔性电极用作锂离子 电池, 极大的提高了锂离子电池的寿命。

\subsection{3 柔性纳米摩擦发电机}

2012年, 中国科学院北京纳米能源与系统研 究所王中林教授 ${ }^{118}$ 发明了摩擦纳米发电机
(TENG), 它可直接收集周围环境中的无处不在随 时发生的各种机械能, 如旋转、敲击、弯曲以及伸 缩等运动, 为电子器件的可持续供电提供了一种 切实可行的解决方案。碳纳米管薄膜能为电极带 来纳米结构, 进而增大了纳米发电机的摩擦接触 面积。Lin 课题组 ${ }^{119}$ 介绍了采用化学气相沉积 (CVD)法制备的一种基于碳纳米管的柔性纳米摩 擦发电机。制造的TENG可以输出 $20 \mathrm{~V}$ 电压, 输出 功率 $10.3 \mathrm{~mW}$, 可以直接驱动多个负载为 $40 \mathrm{mV}$ 绿 色发光二极管(LED)。为了进一步增加纳米发电机 的输出性能, Wang 等 ${ }^{120}$ 研究并实现了一种基于聚 偏氟乙烯 (PVDF) 纳米纤维和聚二甲基硅氧烷 (PDMS)/多壁碳纳米管 (MWCNT)薄膜复合膜的柔 性纳米发电机(图7d), 该器件在摩擦电和压电混合 机制下工作。通过电纺丝工艺形成PVDF纳米纤维 作为压电功能层和摩擦发电层。经测试, 在 $5 \mathrm{~N}$ 的 压力下, 纳米发电机的最大峰值电压为 $25 \mathrm{~V}$, 输出 功率为 $98.56 \mu \mathrm{W}$ 和功率密度为 $1.98 \mathrm{~mW} \cdot \mathrm{cm}^{-3}$ 。

\subsection{4 柔性热电器件}

热电器件能把产生于自然的热能和工业、生 活废热等直接转换为有价值的电能, 实现能源的 循环利用。热能也可用于可穿戴电子设备中关键 部件。在热电模块中, $p$ 型和 $n$ 型热电材料组成的热 电偶是基本工作单元。SWNT由于其出色的电学和 热学性质, 可实现高效的热电能转换 ${ }^{121,122 。 Z h o u ~}$ 
等人 ${ }^{123}$ 最近利用折叠体系结构, 使用悬浮催化剂 CVD法直接生长的单壁碳纳米管薄膜制备了热电 器件 (图7e)。其中 $p$ 型碳纳米管薄膜直接采用了该 课题组制备出的连续网络碳纳米管薄膜, 这是一 种理想柔性热电材料, 拥有优异的 $p$ 型热电功率因 子(室温下最大值为 $\left.2482 \mu \mathrm{W} \cdot \mathrm{m}^{-1} \cdot \mathrm{K}^{-2}\right)$ 。 $n$ 型的碳纳 米管薄膜通过原始的 $p$ 型碳纳米管薄膜用聚醚酰 亚胺 (PEI) 作为 $n$ 型掺杂剂掺杂而成。此方法不仅实 现了高效的电子转移, 使得原始 $p$ 型的碳纳米管薄 膜在无损的情况下迅速转变为 $n$ 型, 同时 PEI的均 匀包覆有效形成了防止 $n$ 型性能退化的保护层。在 温差为 $27.5 \mathrm{~K}$ 时, 这个热电器件能产生约 $2.5 \mu \mathrm{W}$ 的 电力。

\section{3 其他碳纳米管薄膜基柔性电子器件}

碳纳米管薄膜是一种性能优良的柔性导电薄 膜。除了在柔性传感和柔性能源领域的应用外, 还 能用来制备柔性触摸屏、柔性晶体管、柔性光探测 器等。下面主要介绍一下碳纳米管在上述三种其 他柔性电子器件中的应用。

\subsection{1 柔性透明导电材料}

目前, 常用的透明导电薄膜是通过在玻璃祄 底上沉积导电薄膜而制备的。当玻璃的厚度在比 较薄的时候, 导电玻璃能够具备一定的柔性, 可以 发生一定程度上的弯折, 但玻璃本身还是刚性且 易碎的材料, 同时还有制备工艺复杂、价格昂贵等 缺点。由于碳纳米管透明导电薄膜具有良好的导 电性、透光性、柔性且耐弯折以及对湿度和高温稳
定等特点, 该薄膜材料引起了研究者的广泛关注。 在2010年, Zhang等 ${ }^{124}$ 首次使用从超顺排的碳纳米 管垂直阵列中抽出的定向碳纳米管带预埋在 PDMS中, 制备了透明的可拉伸导体, 在重复拉伸 下依然可以保持稳定的电导率(图8a)。Hecht等 125 使用碳纳米管透明导电薄膜组装成触摸屏, 其导 电性甚至优于传统导电玻璃铟锡氧化物(ITO)。该 触摸屏经过 300 万次点触实验和 100 万次笔尖滑动 试验后性能依旧能几乎保持不变, 证明了其良好 的稳定性。清华大学范守善教授课题组 ${ }^{126}$ 基于从 超顺排碳纳米管阵列抽出的薄膜, 演示了一种简 单的、通过直接轧制的技术来制作柔性和可拉伸 的碳纳米管透明导电薄膜的方法(图8b)。使用制备 的碳纳米管导电薄膜组装的实用触摸屏在柔韧性 和耐磨性方面优于以铟锡氧化物(ITO)薄膜为基础 的触摸屏, 并且在导电性方面也不相上下。此碳纳 米管薄膜经适当的激光修整和金属的沉积后，方 块电阻为 $24 \Omega \cdot \mathrm{sq}^{-1}$, 透光率达到了 $83.4 \%$, 具有良 好的性能。该方法目前已经进入市场化阶段, 并且 使柔性触摸屏的实现成为可能。

\subsection{2 柔性晶体管}

硅是传统的半导体材料, 在晶体管领域长久 以来一直占据着主导地位 ${ }^{127-131}$ 。然而业界认为在 2020年左右, 硅材料晶体管的尺寸将无法再缩小, 芯片的性能提升已经接近其物理极限。而且硅晶 体管本身是刚性的器件, 限制了其在柔性可穿戴 领域的发展 ${ }^{132-137}$ 。因此, 人们一直在寻找能够替
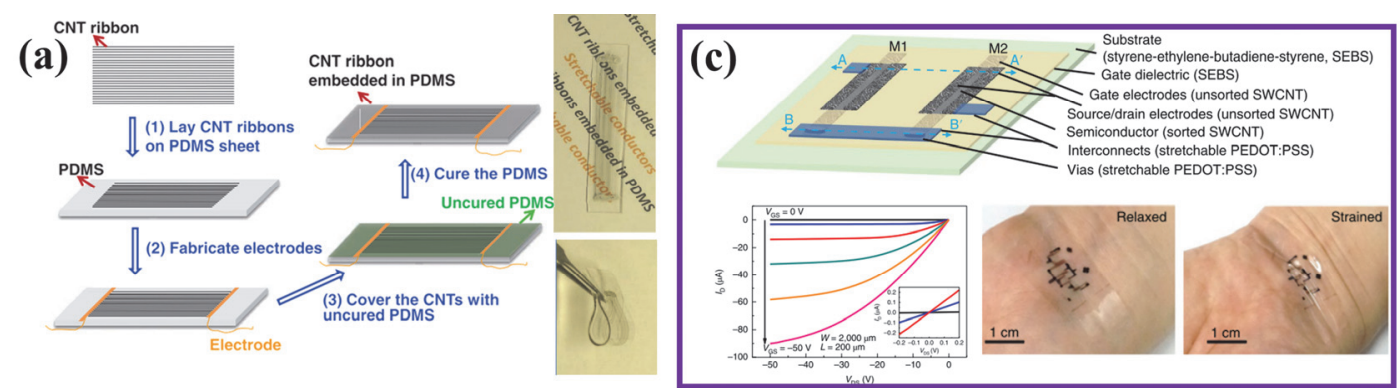

(b)

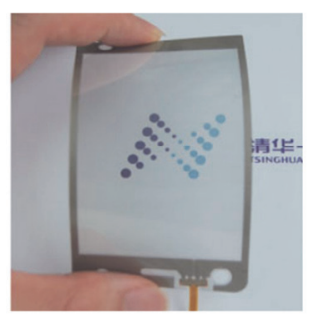

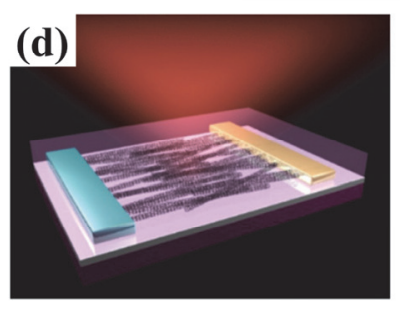

(e)

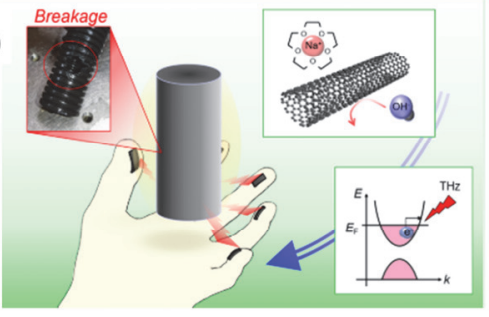

图 8 基于碳纳米管薄膜的其他柔性电子器件

Fig. 8 Other flexible electronic devices based on CNT films.

(a) Schematic illustration of the process to embed CNT ribbons in PDMS and optical images of a CNT/PDMS film on a labeled paper and a folded CNT/PDMS film ${ }^{124}$; (b) Touch panels based on CNT films ${ }^{126}$; (c) Stretchable SWNT TFTs for circuits ${ }^{138}$; (d) Schematic of photodetector based on asymmetrically contacted CNT thin film diode ${ }^{139}$; (e) Fermi-level-controlled flexible and bendable CNT films based terahertz (THz) imagers ${ }^{141}$. 
代当前硅芯片的材料, 碳纳米管就是主要的研究 方向之一 10,138 。北京大学电子学系彭练矛教授团 队成功使用新材料碳纳米管制造出芯片的核心元 器件一晶体管, 其工作速度3倍于英特尔最先进的 $14 \mathrm{~nm}$ 商用硅材料晶体管, 但是能耗只有其四分之 一。Bao课题组 ${ }^{138}$ 探究开发了基于碳纳米管材料的 可拉伸薄膜晶体管(TFTs), 其具有优异的机械和电 学性能(图8c)。该器件以苯乙烯-乙二醇-丁二烯-苯 乙烯(SEBS)氢化弹性体为基体, 采用转移和层合 方法制备了底栅/顶部接触结构的TFTs。其中非极 性SEBS薄膜充当栅极介电层, 将未篎选的单壁碳 纳米管通过光刻技术制作为源-漏极和门极进行 光刻蚀, 将用超分子聚合物篮选出来的半导体性 的SWCNTs作为半导体材料。该器件在扫描电压从 $10 \mathrm{~V}$ 到 $-50 \mathrm{~V}$ 时, 开关比达到了 $10^{4}$ 量级。该器件中 碳纳米管薄膜不仅可以作为晶体管的沟道材料, 也 可以作为电极和互联线材料, 成为具有良好电学 性能的导电薄膜, 而且其中所有材料都有相似的 杨氏模量, 具有很高的拉伸性, 这保证了电路完全 可拉伸, 没有任何应力集中。

\subsection{3 柔性红外探测器}

碳纳米管表现出了强烈的宽带红外线吸收特 性, 而且这可以通过选择不同直径的碳纳米管加 以调节。此外, 由于它们的电子迁移率高, 碳纳米 管的对红外光的响应非常迅速(皮秒级), 所以基于 碳纳米管薄膜的红外探测器迅速成为该领域研究 的热点之一130,134,135,137。早期的碳纳米管红外探测 器基于单根碳纳米管上的对称电极, 其问题在于 碳纳米管的吸收截面非常小, 对红外光的吸收较 弱, 难以得到足够强的信号。为了克服这一缺点, 研究者们使用全半导体的碳纳米管水平阵列薄膜 构建了红外检测器。北京大学彭练矛教授课题组 报道了通过碳纳米管水平阵列制备的红外传感 器, 相同强度光照条件下的光电流比单根碳纳米 管提升 10 倍以上 ${ }^{139}$ (图8d)。此外, 不论是用于望远 镜还是光电通信, 红外探测器必须得到持续的冷 却, 从而避免被杂散热辐射所淹没。2016年, Xie 课题组 ${ }^{140}$ 使用溶液相沉积的碳纳米管薄膜, 通过 不对称电极加工获得了高灵敏度的 “无需冷却” 的红外探测器。因为碳纳米管有特殊的热属性, 在 室温下, 它们自身发出的红外辐射相对极少, 所以 这种新的设计消除了热辐射的影响。另外, 碳纳米 管的导热性能非常好, 因此温度不会在这个探测 器本身上积累。此外, 基于碳纳米管的柔性太赫兹 检测器也被报道。Kawan等 ${ }^{141}$ 开发出基于碳纳米 管薄膜的柔性太赫兹成像仪(图8e)。基于碳纳米管
的柔性太赫兹成像仪放置在手指上, 可以很容易 地包覆手指弯曲的表面。在一个管子中, 通过插入 和旋转粘贴在指尖上的柔性太赫兹成像仪, 可以 清楚地检测到管子的破损。低成本碳纳米管薄膜 的制造方案被开发出来, 例如喷墨涂层等技术, 将 使得大面积的太赫兹成像设备更容易实现。

\section{4 结论与展望}

柔性电子技术是一场全新的电子技术革命, 引 起全世界的广泛关注并得到了迅速发展。美国 Science 杂志将柔性电子技术进展列为 2000 年世界 十大科技成果之一, 与人类基因组草图、克隆技术 等重大发现并列。相对于传统电子, 柔性电子具有 更大的灵活性, 能够在一定程度上适应不同的工 作环境, 满足人类在不同情况下的需求。另外, 随 着社会的飞速发展, 人与信息的有机融合是未来 的发展趋势, 而作为信息载体的各种微电子器件 一旦实现柔性化, 将从本质上促进人与信息的高 效交流。纳米碳材料, 尤其是碳纳米管, 本身是一 种由碳的六元环组成的具有很大长径比的准一维 中空管状纳米材料, 并且具有出色的导电性和导 热性, 优异的机械和化学稳定性以及良好的柔性。 而且微观尺度上单个碳纳米管材料的优越性能可 以扩展到它们的宏观组成体, 如碳纳米管纤维、碳 纳米管薄膜和碳纳米管三维网络等。其中, 碳纳米 管薄膜作为柔性导电薄膜的研究最为成熟, 其多 样化的可控制备方法, 使得碳纳米管薄膜在多种 柔性电子器件领域具有重要的应用潜力和应用 价值。

碳纳米管薄膜主要有两大类制备方法, 分别 是干法制备和湿法制备。其中碳纳米管薄膜的干 法制备中不论是直接从超顺排的碳管阵列直接抽 摸的方法还是通过CVD直接生长的方法, 均已经 能够实现规模化的生产, 且制备出的产品缺陷含 量低、结构完美、性能优异, 能够在柔性电子的制 造中发挥独特的优势。不过, 此方法过程较为繁 琐, 成本较高的缺点亟待解决。而碳纳米管薄膜通 过湿法制备, 不论是应用广泛的真空抽滤法, 还是 可批量化制备的喷涂打印法, 技术的关键都是碳 纳米管的分散问题, 它决定碳纳米管薄膜的成膜 质量及最终薄膜的透明性及导电性。而低成本的 碳纳米管粉体要在溶剂中均匀分散时, 常常都离 不开超声分散, 而碳纳米管在强超声破碎的作用 力下, 常常会因此产生大量缺陷甚至发生断裂, 这 严重影响了后续的应用。所以湿法制备的碳纳米 管薄膜常常存在缺陷含量大、强度较低、电导率低 
等问题。因此, 如何制备有着稳定分散且碳纳米管 保持完好的分散液, 从而提高碳纳米管薄膜的质 量, 也是目前该方法在柔性电子领域做到真正的 精密且规模化应用所面临的问题。

尽管现有技术制备碳纳米管薄膜还有难题要 解决, 面临着诸多的挑战, 但这并不能掩盖它在柔 性电子领域的巨大潜力。高质量的碳纳米管薄膜 由于其本身具有出色的导电性、导热性、机械稳定 性和良好的柔性, 使其能够在各种类型的柔性电 子器件领域都有着广泛的应用。在柔性可穿戴传 感器领域, 碳纳米管薄膜由于它在发生应变时电 阻会改变而可以用作应变传感器; 在碳纳米管薄 膜与高分子弹性体的复合物薄膜受到外界机械力 刺激时, 由于碳纳米管管间的接触会发生变化, 从 而伴随着电阻的变化, 由此可以制作电阻式压力 传感器; 而碳纳米管又作为中空管状一维纳米材 料, 具有丰富的孔隙结构、高表面吸附能力、良好 的导电性、本身的电阻与温度的变化呈线性相关 等性能, 是制作气敏、温敏、湿敏传感器的理想材 料。在柔性能源领域, 碳纳米管的一维结构具有极 大的弯曲半径, 在极度弯曲条件下仍然具有良好 的电化学性能, 所以碳纳米管薄膜作为活性材料 用来同时当做柔性储能器件中的电极和电荷收集 装置; 在纳米摩擦发电机中, 碳纳米管薄膜能为电 极带来纳米结构, 进而增大了纳米发电机的摩擦 接触面积; 由于可以直接制备出 $p$ 型碳纳米管薄 膜, 且可以通过掺杂剂使得其变成 $n$ 型碳纳米管薄 膜, 因此, 碳纳米管薄膜还是一种理想的柔性热电 材料。除此以外, 碳纳米管薄膜作为一种性能优良 的柔性导电薄膜, 在柔性触摸屏、柔性晶体管、柔 性红外探测器等领域也有着非凡的潜力。总之, 改 进生产工艺, 降低成本, 使碳纳米管薄膜的质量能 够进一步提高, 才能使得基于碳纳米管薄膜柔性 电子器件的性能有进一步的突破并能够实现工业 化生产。

综上所述, 碳纳米管薄膜具有优异的电学、光 学和力学特性, 在柔性电子器件领域具有广阔的 应用前景。我们相信, 随着相关技术的进一步成 熟, 基于碳纳米管薄膜材料的柔性电子器件必将 对未来人们的生产和生活方式带来全方位的变 革, 推动信息化和智能化社会的进步和发展。

\section{References}

(1) Britnell, L.; Ribeiro, R. M.; Eckmann, A.; Jalil, R.; Belle, B. D.; Mishchenko, A.; Kim, Y. J.; Gorbachev, R. V.; Georgiou, T.; Morozov, S. V.; et al. Science 2013, 340, 1311. doi: $10.1126 /$ science. 1235547

(2) Eda, G.; Fanchini, G.; Chhowalla, M. Nat. Nanotechnol. 2008, 3, 270. doi: $10.1038 /$ nnano. 2008.83

(3) Gelinck, G. H.; Huitema, H. E. A.; Van Veenendaal, E.; Cantatore, E.; Schrijnemakers, L.; Van der Putten, J.; Geuns, T. C. T.; Beenhakkers, M.; Giesbers, J. B.; Huisman, B. H.; et al. Nat. Mater. 2004, 3, 106. doi: $10.1038 /$ nmat 1061

(4) Kaltenbrunner, M.; Sekitani, T.; Reeder, J.; Yokota, T.; Kuribara, K.; Tokuhara, T.; Drack, M.; Schwoediauer, R.; Graz, I.; Bauer-Gogonea, S.; et al. Nature 2013, 499, 458. doi: 10.1038/nature12314

(5) Mannsfeld, S. C. B.; Tee, B. C. K.; Stoltenberg, R. M.; Chen, C. V. H. H.; Barman, S.; Muir, B. V. O.; Sokolov, A. N.; Reese, C.; Bao, Z. Nat. Mater. 2010, 9, 859. doi: 10.1038/nmat2834

(6) Minemawari, H.; Yamada, T.; Matsui, H.; Tsutsumi, J.; Haas, S.; Chiba, R.; Kumai, R.; Hasegawa, T. Nature 2011, 475, 364. doi: $10.1038 /$ nature 10313

(7) Wu, X. -L.; Wen, T.; Guo, H. -L.; Yang, S.; Wang, X.; Xu, A. -W. ACS Nano 2013, 7, 3589. doi: 10.1021/nn400566d

(8) Xu, Y.; Lin, Z.; Huang, X.; Wang, Y.; Huang, Y.; Duan, X. Adv. Mater. 2013, 25, 5779. doi: 10.1002/adma.201301928

(9) Wang, X.; Liu, J. Micromachines 2016, 7, 206. doi: $10.3390 / \mathrm{mi} 7120206$

(10) Wang, S.; Xu, J.; Wang, W.; Wang, G. -J. N.; Rastak, R.; Molina-Lopez, F.; Chung, J. W.; Niu, S.; Feig, V. R.; Lopez, J.; et al. Nature 2018, 555, 83. doi: 10.1038/nature25494

(11) Takei, K.; Takahashi, T.; Ho, J. C.; Ko, H.; Gillies, A. G.; Leu, P. W.; Fearing, R. S.; Javey, A. Nat. Mater. 2010, 9, 821. doi: $10.1038 /$ nmat 2835

(12) Wu, W.; Wen, X.; Wang, Z. L. Science 2013, 340, 952. doi: $10.1126 /$ science. 1234855

(13) Xu, Y.; Lin, Z.; Huang, X.; Liu, Y.; Huang, Y.; Duan, X. ACS Nano 2013, 7, 4042. doi: 10.1021/nn4000836

(14) Yeo, W. H.; Kim, Y. S.; Lee, J.; Ameen, A.; Shi, L.; Li, M.; Wang, S.; Ma, R.; Jin, S. H.; Kang, Z.; et al. Adv. Mater. 2013, 25, 2773. doi: 10.1002/adma.201204426

(15) Sun, D. -M.; Liu, C.; Ren, W. -C.; Cheng, H. -M. Small 2013, 9, 1188. doi: $10.1002 / \mathrm{smll} .201203154$

(16) Bian, Z.; Song, J.; Webb, R. C.; Bonifas, A. P.; Rogers, J. A.; Huang, Y. $R S C A d v$. 2014, 4, 5694. doi: 10.1039/c3ra45277h

(17) Tortorich, R. P.; Choi, J. -W. Nanomaterials 2013, 3, 453. doi: $10.3390 /$ nano3030453

(18) Xia, K. -L.; Jian, M. -Q.; Zhang, Y. -Y. Acta Phys. -Chim. Sin. 2016, 32, 2427. [夏凯伦, 寒木强, 张荣荣. 物理化学学报, 2016, 32, 2427.] doi: 10.3866/PKU.WHXB201607261

(19) Zhou, Y.; Azumi, R. Sci. Technol. Adv. Mater. 2016, 17, 493. doi: $10.1080 / 14686996.2016 .1214526$ 
(20) Brosseau, C. Surf. Coat. Technol. 2011, 206, 753. doi: 10.1016/j.surfcoat.2011.02.017

(21) Chen, H.; Zeng, S.; Chen, M.; Zhang, Y.; Li, Q. Carbon 2015, 92 , 271. doi: 10.1016/j.carbon.2015.04.010

(22) Chen, K.; Gao, W.; Emaminejad, S.; Kiriya, D.; Ota, H.; Nyein, H. Y. Y.; Takei, K.; Javey, A. Adv. Mater. 2016, 28, 4397. doi: 10.1002/adma.201504958

(23) Luo, M.; Liu, Y.; Huang, W.; Qiao, W.; Zhou, Y.; Ye, Y.; Chen, L. -S. Micromachines 2017, 8, 12. doi: 10.3390/mi8010012

(24) Lin, Y. M.; Appenzeller, J.; Chen, Z. H.; Chen, Z. G.; Cheng, H. M.; Avouris, P. IEEE Electron Device Lett. 2005, 26, 823. doi: 10.1109/led.2005.857704

(25) Lin, Y. M.; Appenzeller, J.; Knoch, J.; Avouris, P. IEEE Trans. Nanotechnol. 2005, 4, 481. doi: 10.1109/tnano.2005.851427

(26) Qiu, C.; Zhang, Z.; Xiao, M.; Yang, Y.; Zhong, D.; Peng, L. -M. Science 2017, 355, 271. doi: 10.1126/science.aaj1628

(27) Li, C. Y.; Chou, T. W. Phys. Rev. B 2005, 71, 235414. doi: 10.1103/PhysRevB.71.235414

(28) Paillet, M.; Michel, T.; Meyer, J. C.; Popov, V. N.; Henrard, L.; Roth, S.; Sauvajol, J. L. Phys. Rev. Lett. 2006, 96, 257401. doi: 10.1103/PhysRevLett.96.257401

(29) Poncharal, P.; Wang, Z. L.; Ugarte, D.; de Heer, W. A. Science 1999, 283, 1513. doi: 10.1126/science.283.5407.1513

(30) Treacy, M. M. J.; Ebbesen, T. W.; Gibson, J. M. Nature 1996, 381, 678. doi: $10.1038 / 381678 \mathrm{a} 0$

(31) Yu, M. F.; Files, B. S.; Arepalli, S.; Ruoff, R. S. Phys. Rev. Lett. 2000, 84, 5552. doi: 10.1103/PhysRevLett.84.5552

(32) Yu, M. F.; Lourie, O.; Dyer, M. J.; Moloni, K.; Kelly, T. F.; Ruoff, R. S. Science 2000, 287, 637. doi: 10.1126/science.287.5453.637

(33) Zhang, R.; Wen, Q.; Qian, W.; Su, D. S.; Zhang, Q.; Wei, F. Adv. Mater. 2011, 23, 3387. doi: 10.1002/adma.201100344

(34) Iijima, S. Nature 1991, 354, 56. doi: 10.1038/354056a0

(35) Bethune, D. S.; Kiang, C. H.; Devries, M. S.; Gorman, G.; Savoy, R.; Vazquez, J.; Beyers, R. Nature 1993, 363, 605. doi: $10.1038 / 363605 \mathrm{a} 0$

(36) Ouyang, M.; Huang, J. L.; Cheung, C. L.; Lieber, C. M. Science 2001, 291, 97. doi: 10.1126/science.291.5501.97

(37) Wildoer, J. W. G.; Venema, L. C.; Rinzler, A. G.; Smalley, R. E.; Dekker, C. Nature 1998, 391, 59. doi: 10.1038/34139

(38) Zhang, R.; Zhang, Y.; Wei, F. Acc. Chem. Res. 2017, 50, 179. doi: $10.1021 /$ acs.accounts.6b00430

(39) Gavillet, J.; Loiseau, A.; Ducastelle, F.; Thair, S.; Bernier, P.; Stephan, O.; Thibault, J.; Charlier, J. C. Carbon 2002, 40, 1649. doi: 10.1016/s0008-6223(02)00007-6

(40) Iijima, S.; Ichihashi, T. Nature 1993, 363, 603. doi: $10.1038 / 363603 \mathrm{a} 0$
(41) Liu, C.; Cong, H. T.; Li, F.; Tan, P. H.; Cheng, H. M.; Lu, K.; Zhou, B. L. Carbon 1999, 37, 1865. doi: 10.1016/s0008-6223(99)00196-7

(42) Zhao, X.; Ohkohchi, M.; Wang, M.; Iijima, S.; Ichihashi, T.; Ando, Y. Carbon 1997, 35, 775. doi: 10.1016/s0008-6223(97)00033-x

(43) Fischer, J. E.; Dai, H.; Thess, A.; Lee, R.; Hanjani, N. M.; Dehaas, D. L.; Smalley, R. E. Phys. Rev. B 1997, 55, R4921. doi: 10.1103/PhysRevB.55.R4921

(44) Guo, T.; Nikolaev, P.; Rinzler, A. G.; Tomanek, D.; Colbert, D. T.; Smalley, R. E. J. Phys. Chem. 1995, 99, 10694. doi: $10.1021 / \mathrm{j} 100027 \mathrm{a} 002$

(45) Kong, J.; Soh, H. T.; Cassell, A. M.; Quate, C. F.; Dai, H. J. Nature 1998, 395, 878. doi: $10.1038 / 27632$

(46) Thess, A.; Lee, R.; Nikolaev, P.; Dai, H. J.; Petit, P.; Robert, J.; Xu, C. H.; Lee, Y. H.; Kim, S. G.; Rinzler, A. G.; et al. Science 1996, 273, 483. doi: $10.1126 /$ science. 273.5274 .483

(47) He, M.; Chernov, A. I.; Fedotov, P. V.; Obraztsova, E. D.; Sainio, J.; Rikkinen, E.; Jiang, H.; Zhu, Z.; Tian, Y.; Kauppinen, E. I.; et al. J. Am. Chem. Soc. 2010, 132, 13994. doi: 10.1021/ja106609y

(48) He, M.; Zhang, S.; Wu, Q.; Xue, H.; Xin, B.; Wang, D.; Zhang, J. Adv. Mater. 2018, e1800805. (in press) doi: 10.1002/adma.201800805

(49) Jiang, K. L.; Li, Q. Q.; Fan, S. S. Nature 2002, 419, 801. doi: $10.1038 / 419801 \mathrm{a}$

(50) Zhang, Y. Y.; Zou, G. F.; Doorn, S. K.; Htoon, H.; Stan, L.; Hawley, M. E.; Sheehan, C. J.; Zhu, Y. T.; Jia, Q. X. ACS Nano 2009, 3, 2157. doi: $10.1021 / \mathrm{nn} 9003988$

(51) Zhang, Y. Y.; Stan, L.; Xu, P.; Wang, H. L.; Doorn, S. K.; Htoon, H.; Zhu, Y. T.; Jia, Q. X. Carbon 2009, 47, 3332. doi: 10.1016/j.carbon.2009.07.056

(52) Xie, H. H.; Zhang, R. F.; Zhang, Y. Y.; Li, P.; Jin, Y. G.; Wei, F. Carbon 2013, 52, 535. doi: 10.1016/j.carbon.2012.10.006

(53) Zhang, R. F.; Zhang, Y. Y.; Zhang, Q.; Xie, H. H.; Qian, W. Z.; Wei, F. ACS Nano 2013, 7, 6156. doi: 10.1021/nn401995z

(54) Zhang, M.; Fang, S. L.; Zakhidov, A. A.; Lee, S. B.; Aliev, A. E.; Williams, C. D.; Atkinson, K. R.; Baughman, R. H. Science 2005, 309, 1215. doi: 10.1126/science. 1115311

(55) Liu, K.; Sun, Y.; Liu, P.; Wang, J.; Li, Q.; Fan, S.; Jiang, K. Nanotechnology 2009, 20, 335705. doi: 10.1088/0957-4484/20/33/335705

(56) Jiang, K.; Wang, J.; Li, Q.; Liu, L.; Liu, C.; Fan, S. Adv. Mater. 2011, 23, 1154. doi: 10.1002/adma.201003989

(57) Ma, W.; Song, L.; Yang, R.; Zhang, T.; Zhao, Y.; Sun, L.; Ren, Y.; Liu, D.; Liu, L.; Shen, J.; et al. Nano Lett. 2007, 7, 2307. doi: $10.1021 / \mathrm{n} 1070915 \mathrm{c}$

(58) Wang, B. -W.; Jiang, S.; Zhu, Q. -B.; Sun, Y.; Luan, J.; Hou, P. -X.; Qiu, S.; Li, Q. -W.; Liu, C.; Sun, D. -M.; et al. Adv. Mater. 2018, 30, 1802057. doi: 10.1002/adma.201802057 
(59) Zhang, D.; Ryu, K.; Liu, X.; Polikarpov, E.; Ly, J.; Tompson, M. E.; Zhou, C. Nano Lett. 2006, 6, 1880. doi: 10.1021/n10608543

(60) Wu, Z. C.; Chen, Z. H.; Du, X.; Logan, J. M.; Sippel, J.; Nikolou, M.; Kamaras, K.; Reynolds, J. R.; Tanner, D. B.; Hebard, A. F.; et al. Science 2004, 305, 1273. doi: 10.1126/science.1101243

(61) He, X.; Gao, W.; Xie, L.; Li, B.; Zhang, Q.; Lei, S.; Robinson, J. M.; Haroz, E. H.; Doorn, S. K.; Wang, W.; et al. Nat. Nanotechnol. 2016, 11, 633. doi: 10.1038/nnano.2016.44

(62) Lima, M. D.; de Andrade, M. J.; Skakalova, V.; Nobre, F.; Bergmann, C. P.; Roth, S. Phys. Status Solidi-Rapid Res. Lett. 2007, 1, 165. doi: $10.1002 /$ pssr.200701086

(63) Ng, M. H. A.; Hartadi, L. T.; Tan, H.; Poa, C. H. P. Nanotechnology 2008, 19, 205703. doi: 10.1088/0957-4484/19/20/205703

(64) Chen, Z.; Yang, Y. L.; Wu, Z. Y.; Luo, G.; Xie, L. M.; Liu, Z. F.; Ma, S. J.; Guo, W. L. J. Phys. Chem. B 2005, 109, 5473. doi: $10.1021 /$ jp045796t

(65) Pei, S.; Du, J.; Zeng, Y.; Liu, C.; Cheng, H. -M. Nanotechnology 2009, 20, 235707. doi: 10.1088/0957-4484/20/23/235707

(66) Engel, M.; Small, J. P.; Steiner, M.; Freitag, M.; Green, A. A.; Hersam, M. C.; Avouris, P. ACS Nano 2008, 2, 2445. doi: $10.1021 / \mathrm{nn} 800708 \mathrm{w}$

(67) Jia, L.; Zhang, Y.; Li, J.; You, C.; Xie, E. J. Appl. Phys. 2008, 104, 074318. doi: $10.1063 / 1.2996033$

(68) Park, H.; Afzali, A.; Han, S. -J.; Tulevski, G. S.; Franklin, A. D.; Tersoff, J.; Hannon, J. B.; Haensch, W. Nat. Nanotechnol. 2012, 7, 787. doi: 10.1038/nnano.2012.189

(69) Cao, Q.; Han, S. -J.; Tulevski, G. S.; Zhu, Y.; Lu, D. D.; Haensch, W. Nat. Nanotechnol. 2013, 8, 180. doi: 10.1038/nnano.2012.257

(70) Joo, Y.; Brady, G. J.; Arnold, M. S.; Gopalan, P. Langmuir 2014, 30, 3460. doi: 10.1021/la500162x

(71) Meitl, M. A.; Zhou, Y. X.; Gaur, A.; Jeon, S.; Usrey, M. L.; Strano, M. S.; Rogers, J. A. Nano Lett. 2004, 4, 1643. doi: $10.1021 / \mathrm{n} 10491935$

(72) Artukovic, E.; Kaempgen, M.; Hecht, D. S.; Roth, S.; GrUner, G. Nano Lett. 2005, 5, 757. doi: 10.1021/n10505254o

(73) Lee, Y. D.; Lee, K. -S.; Lee, Y. -H.; Ju, B. -K. Appl. Surf. Sci. 2007, 254, 513. doi: 10.1016/j.apsusc.2007.06.042

(74) Li, Z.; Kandel, H. R.; Dervishi, E.; Saini, V.; Biris, A. S.; Biris, A. R.; Lupu, D. Appl. Phys. Lett. 2007, 91, 053115. doi: 10.1063/1.2767215

(75) Tenent, R. C.; Barnes, T. M.; Bergeson, J. D.; Ferguson, A. J.; To, B.; Gedvilas, L. M.; Heben, M. J.; Blackburn, J. L. Adv. Mater. 2009, 21, 3210. doi: 10.1002/adma.200803551

(76) Hopkins, A. R.; Straw, D. C.; Spurrell, K. C. Thin Solid Films 2011, 520, 1541. doi: 10.1016/j.tsf.2011.10.043

(77) Carey, T.; Jones, C.; Le Moal, F.; Deganello, D.; Torrisi, F. ACS Appl. Mater. Interfaces 2018, 10, 19948. doi: 10.1021/acsami.8b02784
(78) Kordas, K.; Mustonen, T.; Toth, G.; Jantunen, H.; Lajunen, M.; Soldano, C.; Talapatra, S.; Kar, S.; Vajtai, R.; Ajayan, P. M. Small 2006, 2, 1021. doi: 10.1002/smll.200600061

(79) Fan, Z. J.; Wei, T.; Luo, G. H.; Wei, F. J. Mater. Sci. 2005, 40, 5075. doi: $10.1007 / \mathrm{s} 10853-005-1622-7$

(80) Preston, C.; Song, D.; Dai, J.; Tsinas, Z.; Bavier, J.; Cumings, J.; Ballarotto, V.; Hu, L. Nano Research 2015, 8, 2242. doi: $10.1007 / \mathrm{s} 12274-015-0735-9$

(81) Jian, M. Q.; Xia, K. L.; Wang, Q.; Yin, Z.; Wang, H. M.; Wang, C. Y.; Xie, H. H.; Zhang, M. C.; Zhang, Y. Y. Adv. Funct. Mater. 2017, 27, 1606066 doi: $10.1002 / \mathrm{adfm} .201606066$

(82) Wang, C. Y.; Li, X.; Gao, E. L.; Jian, M. Q.; Xia, K. L.; Wang, Q.; Xu, Z. P.; Ren, T. L.; Zhang, Y. Y. Adv. Mater. 2016, 28, 6640. doi: 10.1002/adma.201601572

(83) Sekitani, T.; Noguchi, Y.; Hata, K.; Fukushima, T.; Aida, T.; Someya, T. Science 2008, 321, 1468. doi: 10.1126/science. 1160309

(84) Zhang, M. C.; Wang, C. Y.; Wang, H. M.; Jian, M. Q.; Hao, X. Y.; Zhang, Y. Y. Adv. Funct. Mater. 2017, 27, 1604795. doi: 10.1002/adfm.201604795

(85) Kang, I.; Schulz, M. J.; Kim, J. H.; Shanov, V.; Shi, D. Smart Mater. Struct. 2006, 15, 737. doi: 10.1088/0964-1726/15/3/009

(86) Thostenson, E. T.; Chou, T. -W. Adv. Mater. 2006, 18, 2837. doi: 10.1002/adma.200600977

(87) Chun, K. -Y.; Oh, Y.; Rho, J.; Ahn, J. -H.; Kim, Y. -J.; Choi, H. R.; Baik, S. Nat. Nanotechnol. 2010, 5, 853. doi: $10.1038 /$ nnano.2010.232

(88) Lipomi, D. J.; Vosgueritchian, M.; Tee, B. C. K.; Hellstrom, S. L.; Lee, J. A.; Fox, C. H.; Bao, Z. Nat. Nanotechnol. 2011, 6, 788. doi: 10.1038/nnano.2011.184

(89) Zhao, H. B.; Zhang, Y. Y.; Bradford, P. D.; Zhou, Q. A.; Jia, Q. X.; Yuan, F. G.; Zhu, Y. T. Nanotechnology 2010, 21, 305502. doi: $10.1088 / 0957-4484 / 21 / 30 / 305502$

(90) Dharap, P.; Li, Z. L.; Nagarajaiah, S.; Barrera, E. V. Nanotechnology 2004, 15, 379. doi: 10.1088/0957-4484/15/3/026

(91) Yamada, T.; Hayamizu, Y.; Yamamoto, Y.; Yomogida, Y.; IzadiNajafabadi, A.; Futaba, D. N.; Hata, K. Nat. Nanotechnol. 2011, 6, 296. doi: $10.1038 /$ nnano.2011.36

(92) Roh, E.; Hwang, B. -U.; Kim, D.; Kim, B. -Y.; Lee, N. -E. ACS Nano 2015, 9, 6252. doi: 10.1021/acsnano.5b01613

(93) Wang, C. Y.; Xia, K. L.; Zhang, M. C.; Jian, M. Q.; Zhang, Y. Y. ACS Appl. Mater. Interfaces 2017, 9, 39484. doi: 10.1021/acsami.7b13356

(94) Xia, K. L.; Wang, C. Y.; Jian, M. Q.; Wang, Q.; Zhang, Y. Y. Nano Research 2018, 11, 1124. doi: 10.1007/s12274-017-1731-z

(95) Park, S.; Kim, H.; Vosgueritchian, M.; Cheon, S.; Kim, H.; Koo, J. H.; Kim, T. R.; Lee, S.; Schwartz, G.; Chang, H.; Bao, Z. Adv. 
Mater. 2014, 26, 7324. doi: 10.1002/adma.201402574

(96) Kim, Y.; Chortos, A.; Xu, W.; Liu, Y.; Oh, J. Y.; Son, D.; Kang, J.; Foudeh, A. M.; Zhu, C.; Lee, Y.; et al. Science 2018, 360, 998. doi: $10.1126 /$ science.aao0098

(97) Jian, M.; Xia, K.; Wang, Q.; Yin, Z.; Wang, H.; Wang, C.; Xie, H.; Zhang, M.; Zhang, Y. Adv. Funct. Mater. 2017, 27, 1606066. doi: 10.1002/adfm.201606066

(98) Woo, C. -S.; Lim, C. -H.; Cho, C. -W.; Park, B.; Ju, H.; Min, D. -H.; Lee, C. -J.; Lee, S. -B. Microelectron. Eng. 2007, 84, 1610. doi: 10.1016/j.mee.2007.01.162

(99) Lin, Z. -D.; Young, S. -J.; Chang, S. -J. IEEE Sensors J. 2015, 15, 7017. doi: $10.1109 /$ jsen.2015.2472968

(100) Hong, S. Y.; Lee, Y. H.; Park, H.; Jin, S. W.; Jeong, Y. R.; Yun, J.; You, I.; Zi, G.; Ha, J. S. Adv. Mater. 2016, 28, 930. doi: 10.1002/adma.201504659

(101) Honda, W.; Harada, S.; Ishida, S.; Arie, T.; Akita, S.; Takei, K. Adv. Mater. 2015, 27, 4674. doi: 10.1002/adma.201502116

(102) Feng, Y.; Cabezas, A. L.; Chen, Q.; Zheng, L. -R.; Zhang, Z. -B. IEEE Sensors J. 2012, 12, 2844. doi: 10.1109/jsen.2012.2202390

(103) Rigoni, F.; Freddi, S.; Pagliara, S.; Drera, G.; Sangaletti, L.; Suisse, J. M.; Bouvet, M.; Malovichko, A. M.; Emelianov, A. V.; Bobrinetskiy, I. I. Nanotechnology 2017, 28, 255502. doi: 10.1088/1361-6528/aa6da7

(104) Tai, Y.; Lubineau, G. Small 2017, 13, 1603486. doi: $10.1002 / \mathrm{smll} .201603486$

(105) Zhao, H.; Zhang, T.; Qi, R.; Dai, J.; Liu, S.; Fei, T. ACS Appl. Mater. Interfaces 2017, 9, 28002. doi: 10.1021/acsami.7b05181

(106) Wang, H.; Wang, C.; Jian, M.; Wang, Q.; Xia, K.; Yin, Z.; Zhang, M.; Liang, X.; Zhang, Y. Nano Research 2018, 11, 2347. doi: $10.1007 / \mathrm{s} 12274-017-1782-1$

(107) Chen, P. -C.; Shen, G.; Sukcharoenchoke, S.; Zhou, C. Appl. Phys. Lett. 2009, 94, 043133. doi: 10.1063/1.3069277

(108) Pan, S.; Lin, H.; Deng, J.; Chen, P.; Chen, X.; Yang, Z.; Peng, H. Adv. Energy Mater. 2015, 5, 1401438 . doi: 10.1002/aenm.201401438

(109) Kaempgen, M.; Chan, C. K.; Ma, J.; Cui, Y.; Gruner, G. Nano Lett. 2009, 9, 1872. doi: 10.1021/n18038579

(110) Hu, S.; Rajamani, R.; Yu, X. Appl. Phys. Lett. 2012, 100, 104103. doi: $10.1063 / 1.3691948$

(111) Xi, S.; Kang, Y.; Qu, S.; Han, S. Mater. Lett. 2016, 175, 126. doi: 10.1016/j.matlet.2016.03.143

(112) Fang, X.; Shen, C.; Ge, M.; Rong, J.; Liu, Y.; Zhang, A.; Wei, F.; Zhou, C. Nano Energy 2015, 12, 43. doi: 10.1016/j.nanoen.2014.11.052

(113) Kang, C.; Cha, E.; Baskaran, R.; Choi, W. Nanotechnology 2016, 27, 105402. doi: 10.1088/0957-4484/27/10/105402
(114) Sun, X.; Liu, Z.; Li, N.; Wu, X.; Nie, Y.; Pang, Z.; Yue, L.; Tang, H. Nano 2016, 11, 1650120. doi: 10.1142/s1793292016501204

(115) Kim, S.; Song, H.; Jeong, Y. Carbon 2017, 113, 371. doi: 10.1016/j.carbon.2016.11.019

(116) Chew, S. Y.; Ng, S. H.; Wang, J.; Novak, P.; Krumeich, F.; Chou, S. L.; Chen, J.; Liu, H. K. Carbon 2009, 47, 2976. doi: 10.1016/j.carbon.2009.06.045

(117) Lee, S.; Song, H.; Hwang, J. Y.; Jeong, Y. Fib. Polym. 2017, 18, 2334. doi: 10.1007/s12221-017-7715-5

(118) Cheng, J.; Ding, W.; Zi, Y.; Lu, Y.; Ji, L.; Liu, F.; Wu, C.; Wang, Z. L. Nat. Commun. 2018, 9, 3733. doi: 10.1038/s41467-018-06198-x

(119) Khan, S. A.; Zhang, H. L.; Xie, Y.; Gao, M.; Shah, M. A.; Qadir, A.; Lin, Y. Adv. Eng. Mater. 2017, 19, 1600710. doi: 10.1002/adem.201600710

(120) Wang, X.; Yang, B.; Liu, J.; Zhu, Y.; Yang, C.; He, Q. Sci. Rep. 2016, 6, 36409. doi: 10.1038/srep36409

(121) Kim, S. L.; Choi, K.; Tazebay, A.; Yu, C. ACS Nano 2014, 8, 2377. doi: $10.1021 / \mathrm{nn} 405893 \mathrm{t}$

(122) Blackburn, J. L.; Ferguson, A. J.; Cho, C.; Grunlan, J. C. Adv. Mater. 2018, 30, 1704386. doi: 10.1002/adma.201704386

(123) Zhou, W.; Fan, Q.; Zhang, Q.; Cai, L.; Li, K.; Gu, X.; Yang, F.; Zhang, N.; Wang, Y.; Liu, H.; Zhou, W.; Xie, S. Nat. Commun. 2017, 8, 14886. doi: $10.1038 /$ ncomms 14886

(124) Zhang, Y. Y.; Sheehan, C. J.; Zhai, J. Y.; Zou, G. F.; Luo, H. M.; Xiong, J.; Zhu, Y. T.; Jia, Q. X. Adv. Mater. 2010, 22, 3027. doi: 10.1002/adma.200904426

(125) Hecht, D. S.; Thomas, D.; Hu, L.; Ladous, C.; Lam, T.; Park, Y.; Irvin, G.; Drzaic, P. J. Soc. Inform. Display 2009, 17, 941. doi: $10.1889 /$ jsid17.11.941

(126) Feng, C.; Liu, K.; Wu, J. -S.; Liu, L.; Cheng, J. -S.; Zhang, Y.; Sun, Y.; Li, Q.; Fan, S.; Jiang, K. Adv. Funct. Mater. 2010, 20, 885. doi: 10.1002/adfm.200901960

(127) Fan, S. S.; Chapline, M. G.; Franklin, N. R.; Tombler, T. W.; Cassell, A. M.; Dai, H. J. Science 1999, 283, 512. doi: $10.1126 /$ science. 283.5401 .512

(128) Yao, Z.; Kane, C. L.; Dekker, C. Phys. Rev. Lett. 2000, 84, 2941. doi: 10.1103/PhysRevLett.84.2941

(129) Duan, X. F.; Huang, Y.; Cui, Y.; Wang, J. F.; Lieber, C. M. Nature 2001, 409, 66. doi: 10.1038/35051047

(130) Bachilo, S. M.; Strano, M. S.; Kittrell, C.; Hauge, R. H.; Smalley, R. E.; Weisman, R. B. Science 2002, 298, 2361. doi: $10.1126 /$ science. 1078727

(131) Baughman, R. H.; Zakhidov, A. A.; de Heer, W. A. Science 2002, 297, 787. doi: 10.1126/science. 1060928

(132) Gudiksen, M. S.; Lauhon, L. J.; Wang, J.; Smith, D. C.; Lieber, C. M. Nature 2002, 415, 617. doi: 10.1038/415617a 
(133) Lee, C. J.; Lee, T. J.; Lyu, S. C.; Zhang, Y.; Ruh, H.; Lee, H. J. Appl. Phys. Lett. 2002, 81, 3648. doi: 10.1063/1.1518810

(134) Freitag, M.; Martin, Y.; Misewich, J. A.; Martel, R.; Avouris, P. H. Nano Lett. 2003, 3, 1067. doi: 10.1021/n1034313e

(135) Levitsky, I. A.; Euler, W. B. Appl. Phys. Lett. 2003, 83, 1857. doi: $10.1063 / 1.1606099$

(136) Xia, Y. N.; Yang, P. D.; Sun, Y. G.; Wu, Y. Y.; Mayers, B.; Gates, B.; Yin, Y. D.; Kim, F.; Yan, Y. Q. Adv. Mater. 2003, 15, 353. doi: 10.1002/adma.200390087

(137) Qiu, X. H.; Freitag, M.; Perebeinos, V.; Avouris, P. Nano Lett. 2005, 5, 749. doi: $10.1021 / \mathrm{n} 1050227 \mathrm{y}$

(138) Zhu, C.; Chortos, A.; Wang, Y.; Pfattner, R.; Lei, T.; Hinckley, A. C.;
Pochorovski, I.; Yan, X.; To, J. W. F.; Oh, J. Y.; et al. Nat. Electron. 2018, 1, 183. doi: 10.1038/s41928-018-0041-0

(139) Liu, Y.; Wei, N.; Zeng, Q.; Han, J.; Huang, H.; Zhong, D.; Wang, F.; Ding, L.; Xia, J.; Xu, H.; et al. Adv. Opt. Mater. 2016, 4, 238. doi: 10.1002/adom.201500529

(140) Zeng, Q. S.; Wang, S.; Yang, L. J.; Wang, Z. X.; Pei, T.; Zhang, Z. Y.; Peng, L. M.; Zhou, W. W.; Liu, J.; Zhou, W. Y.; et al. Opt. Mater. Express 2012, 2, 839. doi: 10.1364/Ome.2.000839

(141) Suzuki, D.; Ochiai, Y.; Nakagawa, Y.; Kuwahara, Y.; Saito, T.; Kawano, Y. ACS Appl. Nano Mater. 2018, 1, 2469. doi: $10.1021 /$ acsanm. $8 \mathrm{~b} 00421$ 